\title{
NÉRON MODELS OF ALGEBRAIC CURVES
}

\author{
QING LIU AND JILONG TONG \\ Dedicated to Michel Raynaud on the occasion of his seventy-fifth birthday
}

\begin{abstract}
Let $S$ be a Dedekind scheme with field of functions $K$. We show that if $X_{K}$ is a smooth connected proper curve of positive genus over $K$, then it admits a Néron model over $S$, i.e., a smooth separated model of finite type satisfying the usual Néron mapping property. It is given by the smooth locus of the minimal proper regular model of $X_{K}$ over $S$, as in the case of elliptic curves. When $S$ is excellent, a similar result holds for connected smooth affine curves different from the affine line, with locally finite type Néron models.
\end{abstract}

\section{INTRODUCTION}

In 1964, A. Néron [11] introduced the notion of Néron models (see Definition 2.2) of abelian varieties over the fraction field of a Dedekind domain, and proved the existence of these models (see the introduction of [1] for a detailed presentation). Since then, this notion has been generalized to smooth commutative algebraic groups and to torsors under these groups (see [1], §6.5). In this work we investigate the case of smooth proper or affine curves.

Let $S$ be a Dedekind scheme, that is, a noetherian regular connected scheme of dimension 1. Let $K=K(S)$ be its field of functions. Let $X_{K}$ be a smooth connected curve over $K$. When $X_{K}$ is proper of positive genus, a canonical smooth model is the smooth locus $X_{\mathrm{sm}}$ of the minimal proper regular model of $X_{K}$ over $S$. When $X_{K}$ is an elliptic curve, it is well known that $X_{\mathrm{sm}}$ is the Néron model of $X_{K}$ (see [11] or [8], 10.2.14). The first main result of this work is a generalization of the latter fact to higher genus.

Theorem 1.1. (Theorem 4.1) Let $X_{K}$ be a proper smooth connected curve of positive genus over $K$. Then the smooth locus $X_{\mathrm{sm}}$ of the minimal proper regular model of $X_{K}$ over $S$ is the Néron model of $X_{K}$.

Date: December 18, 2013.

2000 Mathematics Subject Classification. 14H25, 14G20, 14G40, 11G35.

Key words and phrases. Néron model, curve, good reduction. 
When $S$ is excellent, we actually prove a slightly more general result: for a regular proper connected curve $X_{K} / K$ of positive arithmetic genus, the smooth locus of $X_{K}$ admits a Néron model over $S$, equal to the smooth locus of the minimal proper regular model of $X_{K}$ over $S$.

As an immediate consequence of Theorem 1.1, we have the next corollary.

Corollary 1.2. Let $X_{K}$ be as in Theorem 1.1. Let $Y$ be a smooth scheme over $S$ and let $f_{K}: Y_{K} \rightarrow X_{K}$ be a morphism of $K$-schemes. Then

(1) the morphism $f_{K}$ extends uniquely to a morphism of S-schemes $Y \rightarrow X_{\mathrm{sm}}$

(2) (Corollary 4.7) if $Y$ is proper over $S$ (i.e., $Y_{K}$ has good reduction over $S$ ) and $f_{K}$ is dominant, then $X_{\mathrm{sm}}$ is proper over $S$ and $X_{K}$ has good reduction over $S$.

In the second part of this paper ( $\$ 5-\$ 7)$, we consider Néron lft-models (Néron model locally of finite type) of smooth affine curves. The main result of this second part is

Theorem 1.3. (Theorem 7.10) Suppose $S$ is excellent. Let $U_{K}$ be an affine smooth and geometrically connected curve over $K$, not isomorphic to $\mathbb{A}_{K}^{1}$. Then $U_{K}$ admits a Néron lft-model $U$ over $S$.

Note that in general, the scheme $U$ in the above theorem is not of finite type. However, necessary and sufficient conditions (in terms of the points at infinity of $U_{K}$ ) can be found in Proposition 7.11 to insure that $U$ is of finite type over $S$.

The paper is organized as follows. Some basic properties of Néron lft-models are assembled in \$2, In \$3, we prove a crucial technical result (Proposition 3.8) on the image of a morphism $f: Y \rightarrow X$ from a smooth $S$-scheme to a normal relative curve over $S$. In $₫ 4$, we prove the main theorem 4.1 on the existence of Néron models for proper smooth curves of positive genus. In \$5, we study the Néron lft-models of open subschemes of a curve having already a Néron lft-model (Theorem 5.1). \$6 is devoted to the existence of the Néron model of some special affine open subschemes of a smooth conic over local Dedekind schemes $S$. Finally we prove Theorem 1.3 in $\$ 7$.

The present work grew from a partial answer to a question asked by an anonymous poster at mathoverflow.net/questions/110359/, on the existence of Néron models of projective curves. We would like to thank A. Javanpeykar and M. Raynaud for their interests in this work and especially for their comments which improve the presentation of the paper. 
Notation: In all this paper, unless explicitly mentioned, the letter $S$ denotes a Dedekind scheme (that is, a noetherian regular connected scheme of dimension 1), $K$ denotes its field of functions $K(S)$. Symbols such as $X_{K}, Y_{K}, U_{K}$ usually denote a scheme over $K$. On the other hand, for any $S$-scheme $X, X_{K}$ also denotes the generic fiber of $X$.

\section{BASIC PROPERTIES}

Let $S, K$ be as above.

Definition 2.1 Let $X_{K}$ be a separated algebraic variety (i.e. separated scheme of finite type) over $K$. A model of $X_{K}$ over $S$ is a locally finite type, separated and flat1 scheme over $S$ endowed with an isomorphism from its generic fiber to $X_{K}$.

Definition $2.2\left([1]\right.$ 10.1/1, 1.2/1) Let $X_{K}$ be a separated smooth algebraic variety over $K$. A Néron lft-model of $X_{K}$ over $S$ or an $S$ Néron lft-model of $X_{K}$ is a smooth model $X$ of $X_{K}$ over $S$ satisfying the following universal property, called Néron mapping property:

for any smooth scheme $Y \rightarrow S$, the canonical map (once the generic fiber of $X$ is identified with $X_{K}$ )

$$
\operatorname{Mor}_{S}(Y, X) \rightarrow \operatorname{Mor}_{K}\left(Y_{K}, X_{K}\right)
$$

is a bijection.

A Néron lft-model of finite type is called a Néron model.

Remark 2.3 (1) The universal property above implies the uniqueness (up to a unique isomorphism) of the Néron lft-model if it exists.

(2) Let $X$ be a model of $X_{K}$. As $X$ is separated, the map (2.1) is always injective. So it is enough to check the surjectivity for the Néron mapping property. By the injectivity, when $S$ is local, it is also enough to check the surjectivity with $Y$ smooth of finite type having irreducible fibers over $S$.

(3) Let $X_{K}$ be a separated smooth algebraic variety over $K$. If each connected component of $X_{K}$ admits an $S$-Néron lft-model, then $X_{K}$ has also an $S$-Néron lft-model given by the disjoint union of the $S$-Néron lft-models of the connected components. This holds similarly for Néron models.

Proposition 2.4. Let $X$ be an $S$-model of $X_{K}$. Let $S^{\prime} \rightarrow S$ be a morphism of Dedekind schemes. Denote by $K^{\prime}$ the function field of $S^{\prime}$.

\footnotetext{
${ }^{1}$ In this work, we do not require the models to be faithfully flat.
} 
(1) Assume that the morphism $S^{\prime} \rightarrow S$ is faithfully flat, and that $X_{S^{\prime}}:=$ $X \times_{S} S^{\prime}$ is the Néron lft-model (resp. Néron model) of $X_{K^{\prime}}:=$ $X \times_{\operatorname{Spec}(K)} \operatorname{Spec}\left(K^{\prime}\right)$ over $S^{\prime}$. Then $X$ is the Néron lft-model (resp. Néron model) of $X_{K}$ over $S$.

(2) Assume that $S^{\prime} \rightarrow S$ can be written as a filtered inverse limit of affine smooth schemes of finite type over $S$, and that $X$ is the Néron lft-model (resp. Néron model) of $X_{K}$ over $S$. Then the base change $X_{S^{\prime}}$ is the Néron lft-model (resp. Néron model) of $X_{K^{\prime}}$ over $S^{\prime}$.

(3) Assume that $S^{\prime} \rightarrow S$ is an extension of local Dedekind schemes of ramification index $1^{2}$ with $S$ excellent, and that $X$ is the Néron lft-model over $S$. Then, $X_{S^{\prime}}$ is the Néron lft-model over $S^{\prime}$.

Proof. (1) This is a direct application of fpqc descent, so we omit the details here.

(2) Let $Y^{\prime}$ be a smooth $S^{\prime}$-scheme, and let $f_{K^{\prime}}: Y_{K^{\prime}}^{\prime} \rightarrow X_{K^{\prime}}$ be a morphism of $K^{\prime}$-schemes. Without loss of generality, we may and do assume that the scheme $Y^{\prime}$ is quasi-compact, hence is of finite presentation over $S^{\prime}$. Consequently, the $S^{\prime}$-scheme $Y^{\prime}$ (resp. the morphism $f_{K^{\prime}}: Y_{K^{\prime}}^{\prime} \rightarrow X_{K^{\prime}}$ ) descends to an $S_{0^{-}}$-scheme $Y_{0}$ (resp. to a morphism of

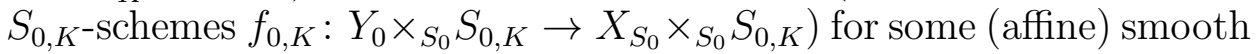
morphism $S_{0} \rightarrow S([5]$, IV 8.8.2). Thus, we only need to prove that the morphism $f_{0, K}$ can be extended to a morphism $f_{0}: Y_{0} \rightarrow X \times{ }_{S} S_{0}$. Consider the composite $Y_{0} \rightarrow S_{0} \rightarrow S$, which defines a smooth $S$-scheme. The morphism $f_{0, K}$ gives a morphism of $K$-schemes from $Y_{0} \times{ }_{S} \operatorname{Spec}(K)$ to $X_{K}$. As $X$ is the $S$-Néron lft-model of $X_{K}$, the Néron mapping property implies a morphism of $S$-schemes $g: Y_{0} \rightarrow X$ making the following diagram commutative:

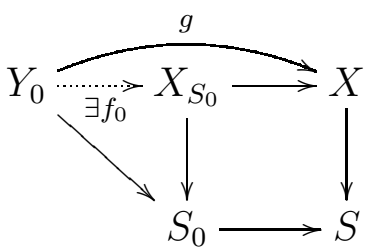

We obtain a morphism $f_{0}: Y_{0} \rightarrow X_{S_{0}}$ of $S_{0^{-}}$schemes extending $f_{0, K}$, as required. If $X$ is the $S$-Néron model of $X_{K}$, then $X_{S^{\prime}}$ is of finite type over $S^{\prime}$ and is the $S^{\prime}$-Néron model of $X_{K^{\prime}}$.

(3) Write $S=\operatorname{Spec}(R)$ and $S^{\prime}=\operatorname{Spec}\left(R^{\prime}\right)$. As $S$ is excellent, by [1], 3.6/2, the morphism $R \rightarrow R^{\prime}$ is regular. Consequently, by a result of Néron ([1], 3.6/8, see also [12], 2.5 for a more general statement),

\footnotetext{
${ }^{2}$ see [1], 3.6/1 for the definition, the residue extension is required to be (not necessarily algebraic) separable.
} 
$R^{\prime}$ can be written as a filtered inductive limit of smooth $R$-algebras of finite type. Therefore, $X_{S^{\prime}}$ is the Néron lft-model over $S^{\prime}$ by (2).

Corollary 2.5. Let $S$ be a Dedekind scheme with field of functions $K$, and let $X$ be an $S$-scheme locally of finite type (resp. an S-scheme of finite type) with smooth finite type generic fiber $X_{K}$. Then $X$ is the $S$-Néron lft-model of $X_{K}$ (resp. S-Néron model of $\left.X_{K}\right)$ if and only if for any closed point $s \in S, X \times{ }_{S} \operatorname{Spec}\left(\mathcal{O}_{S, s}\right)$ is the $\operatorname{Spec}\left(\mathcal{O}_{S, s}\right)$-Néron lft-model of $X_{K}$ (resp. $\operatorname{Spec}\left(\mathcal{O}_{S, s}\right)$-Néron model of $\left.X_{K}\right)$.

Proof. If $X / S$ is of finite type, then this corollary is proved in [1], $1.2 / 4$. The same proof applies in the locally finite type case. For the convenience of the readers, we reproduce the proof in this case here. The direct implication follows from Proposition 2.4 (2). Conversely, if $X \times_{S} \operatorname{Spec}\left(\mathcal{O}_{S, s}\right)$ is the Néron lft-model of $X_{K} \operatorname{over} \operatorname{Spec}\left(\mathcal{O}_{S, s}\right)$ for any closed point $s \in S$, it follows that $X / S$ is smooth and separated ([5], IV.8.10.5(v)). It remains to check the Néron mapping property for $X / S$ (Definition 2.2). Consider $Y$ a smooth $S$-scheme, and $f_{K}: Y_{K} \rightarrow X_{K}$ a morphism of $K$-schemes, we need to extend $f_{K}$ to a morphism of $S$-schemes from $Y$ to $X$. We may assume that $Y$ is quasi-compact, hence is of finite presentation over $S$. Now, for any closed point $s \in S$, our assumptions imply that one can find an extension $f_{\mathcal{O}_{S, s}}$ of $f_{K}$ over $\operatorname{Spec}\left(\mathcal{O}_{S, s}\right)$. As $Y / S$ is of finite presentation and $X / S$ is locally of finite presentation, we may descend $f_{\mathcal{O}_{S, s}}$ to a morphism $f_{s}: Y \times_{S} V \rightarrow$ $X \times{ }_{S} V$ over some open neighborhood $V \subseteq S$ of $s$ ([5], IV 8.8.2). As $X / S$ is separated, and $Y / S$ is flat, these local extensions of $f_{K}$ are compatible with each other. As a result, they can be glued together to a morphism $f: Y \rightarrow S$ extending $f_{K}$, as desired.

The next lemma says that we can restrict ourselves to geometrically connected varieties $X_{K}$ without loss of generality. Denote by $\bar{K}$ an algebraic closure of $K$. For any closed point $s \in S$ we denote by $K_{s}^{\text {sh }}$ the fraction field of the strict henselization of the local ring $\mathcal{O}_{S, s}$.

Lemma 2.6. Let $X_{K}$ be a separated smooth connected variety over $K$. Let $K^{\prime}=K\left(X_{K}\right) \cap \bar{K}$ be the field of constants of $K\left(X_{K}\right)$, let $S^{\prime}$ be the integral closure of $S$ in $K^{\prime}$ and let $T \subseteq S^{\prime}$ be the étale locus of $S^{\prime} \rightarrow S$. Then

(1) $X_{K}$ is canonically a separated smooth and geometrically connected variety over $K^{\prime}$;

(2) $X_{K} / K$ admits an $S$-Néron lft-model (resp. S-Néron model) if and only if $\operatorname{dim} T=0$, or $\operatorname{dim} T=1$ and $X_{K} / K^{\prime}$ admits a $T$-Néron lft-model (resp. T-Néron model). 
Proof. (1) As $K^{\prime} \subseteq \mathcal{O}_{X_{K}}\left(X_{K}\right) \subseteq K\left(X_{K}\right)$ by the normality of $X_{K}$, the latter has a canonical structure of $K^{\prime}$-variety. As $K^{\prime}$ is algebraically closed in $K\left(X_{K}\right), X_{K} / K^{\prime}$ is geometrically connected. As $X_{K} \times_{\operatorname{Spec}\left(K^{\prime}\right)}$ $\operatorname{Spec}(\bar{K})$ is a connected component of $X_{K} \times_{\operatorname{Spec}(K)} \operatorname{Spec}(\bar{K}), X_{K} / K^{\prime}$ is separated and smooth.

(2) Note that because $X_{K}$ is smooth over $K, K^{\prime} / K$ is separable, so $S^{\prime} \rightarrow S$ is finite. If $\operatorname{dim} T=0$, then $S$ is semi-local and $S^{\prime} \rightarrow S$ is ramified at all closed points. This implies that for all closed points $s \in S, X_{K}$ has no $K_{s}^{\text {sh }}$-point, so $X_{K}$ is its own $S$-Néron model. Suppose now that $\operatorname{dim} T=1$ and that $X_{K} / K^{\prime}$ has a $T$-Néron lft-model $X$. Then the composition with $T \rightarrow S$ makes $X$ into a smooth separated $S$-scheme, with generic fiber $X_{K} / K$. Let us check that it satisfies the Néron mapping property. Let $Y \rightarrow S$ be a smooth scheme and let $f_{K}: Y_{K} \rightarrow X_{K}$ be a $K$-morphism. Then $Y_{K} \rightarrow \operatorname{Spec}(K)$ also factors through $Y_{K} \rightarrow \operatorname{Spec}\left(K^{\prime}\right)$ via $X_{K} \rightarrow \operatorname{Spec}\left(K^{\prime}\right)$. In particular, $f_{K}$ is a $K^{\prime}$-morphism. On the other hand, as $Y$ is normal, $Y \rightarrow S$ factors through $Y \rightarrow S^{\prime}$. The latter has image in $T$ by Corollary 3.2 and makes $Y$ a smooth $T$-scheme. So $f_{K}$ extends to a $T$-morphism $f: Y \rightarrow X$, which is a fortiori an $S$-morphism.

Conversely, if $X_{K} / K$ has an $S$-Néron lft-model $X$, the above arguments show that $X$ is canonically a smooth separated $T$-scheme, and the generic fiber of $X_{T}$ is nothing but $X_{K}$ viewed as a scheme over $K^{\prime}$. If $\operatorname{dim} T=1$, the Néron mapping property of $X \rightarrow T$ is immediate to verify.

Finally, as $T \rightarrow S$ is of finite type and separated, $X \rightarrow T$ is of finite type if and only if $X \rightarrow S$ is of finite type.

Corollary 2.7. Let $X_{K}$ be a smooth $K$-variety of dimension zero. Then $X_{K}$ admits a Néron model over $S$.

Proof. By Proposition 2.3 (3) and Lemma 2.6, we can suppose $X_{K} / K$ is geometrically connected, smooth of dimension 0 . Then $X_{K}=\operatorname{Spec}(K)$, and $S$ is clearly the Néron model of $X_{K}$ over $S$.

The following proposition will be used to see when a Néron lft-model is a Néron model (e.g. in Proposition [7.11).

Proposition 2.8. Let $X \rightarrow S$ be a separated morphism locally of finite type, such that $X \times{ }_{S} \operatorname{Spec}\left(\mathcal{O}_{S, s}\right)$ is of finite type for all $s$.

(1) If $X$ is irreducible and $X_{K}$ is proper over $K$, then $X$ is of finite type over $S$.

(2) If $X_{K}$ is affine, $X_{s}$ is irreducible for all $s \in S$ and $S$ is excellent, then $X$ is of finite type over $S$. 
(3) If $X$ is of finite type and if $X_{K} / K$ is geometrically connected, then $X_{s}$ is geometrically connected for all $s$ in some dense open subset of $S$.

Proof. (1) Let $U$ be a quasi-compact open subset of $X$ such that $X_{K} \subseteq$ $U$, and let $U^{\prime}$ be a Nagata compactification of $U \rightarrow S$. Then $U$ and $U^{\prime}$ are of finite type over $S$ sharing the same generic fiber. So there exists a dense open subset $V$ of $S$ such that $U \times_{S} V \cong U^{\prime} \times_{S} V$ is proper over $V$. The inclusion $U \times_{S} V \rightarrow X \times_{S} V$ is then open and closed. As $X \times_{S} V$ is irreducible, $X \times_{S} V=U \times_{S} V$, thus is of finite type over $V$. The remaining part $X \times_{S}(S \backslash V)$ is a finite union of quasi-compact subsets, so $X$ is quasi-compact.

(2) As in (1), to prove $X$ is quasi-compact, we are allowed to shrink $S$. Let $U$ be a quasi-compact open subset of $X$ containing $X_{K}$, and let $W$ be an affine finite type $S$-scheme such that $W_{K}=X_{K}$. As $U, W$ are of finite type over the noetherian scheme $S$ with the same generic fiber, shrinking $S$ if necessary, we can suppose that $U=W$ is affine, and that $U \rightarrow S$ is surjective (with $S$ affine). We claim that $X=U$. Let $U^{\prime}$ be any affine open subset of $X$ not contained in $U$ and $F:=U^{\prime} \backslash\left(U \cap U^{\prime}\right)$. Then $F \neq \emptyset$, so it has pure codimension 1 in $U^{\prime}$ because $U \cap U^{\prime}$ is affine (see [8], Exercise 4.1.15. The hypothesis $S$ excellent implies that the normalization map of $U^{\prime}$ is finite). As $F_{K}=\emptyset, F$ is then a finite union of vertical divisors, which is impossible since $U_{s}$ is dense in $X_{s}$. Consequently, $X=U$ is of finite type over $S$.

(3) This follows from [5], IV.9.7.7 after noticing that, as $S$ is irreducible, a dense locally constructible subset of $S$ contains an open dense subset.

Remark 2.9 In general, the condition $X \times_{S} \operatorname{Spec}\left(\mathcal{O}_{S, s}\right)$ of finite type over $\mathcal{O}_{S, s}$ for all $s$ is not sufficient to conclude that $X$ is of finite type over $S$, as an example of Oesterlé ([1], 10.1/11) shows. See also Remark 7.12 .

\section{Image of SMoOTh SCHEMES}

Let $f: Y \rightarrow X$ be a morphism of $S$-schemes. In this section, we study geometric properties of $X$ at the points of $f(Y)$, when $Y$ is smooth over $S$. The main result is Proposition 3.6 which states that $X$ is smooth at points of $f(Y)$ under some mild hypothesis. Its Corollary 3.8 is a principal ingredient of the proof of Theorem 4.1.

Let $Y$ be a scheme. For any morphism locally of finite type $Z \rightarrow Y$, we denote by $\operatorname{sm}(Z / Y) \subseteq Z$ the smooth locus of $Z \rightarrow Y$. This is an open subset of $Z$ if $Y$ is locally noetherian. 
Lemma 3.1. Let $Z \rightarrow Y$ be a morphism locally of finite type. Suppose that $Y, Z$ are locally noetherian and regular. Then for any section $\sigma: Y \rightarrow Z$, the image $\sigma(Y)$ is contained in the smooth locus $\operatorname{sm}(Z / Y)$ of $Z \rightarrow Y$.

Proof. See [1], 3.1/2.

Corollary 3.2. Let $S$ be a locally noetherian regular scheme. Let $f: Y \rightarrow X$ be a morphism between two $S$-schemes locally of finite type. Suppose that $Y$ is smooth over $S$, and that $X$ is regular. Then $f(Y)$ is contained in the smooth locus of $X \rightarrow S$.

Proof. Consider the $Y$-scheme $Z:=X \times{ }_{S} Y$. Notice that $Z$ is regular, being smooth over $X$. The morphism $f$ induces a section $\sigma: Y \rightarrow Z$, $y \mapsto(f(y), y)$, of the second projection $Z \rightarrow Y$. By Lemma 3.1,

$$
\sigma(Y) \subseteq \operatorname{sm}(Z / Y)=\operatorname{sm}(X / S) \times{ }_{S} Y,
$$

hence $f(Y) \subseteq \operatorname{sm}(X / S)$.

Corollary 3.2 does not hold in general if we remove the regularity hypothesis on $X$. However, in the situation of relative curves, we can weaken the regularity hypothesis to the normality of $X$ (Proposition 3.6). We first prove some preliminary results.

Lemma 3.3. Let $S$ be an irreducible locally noetherian scheme, and let $X, Y$ be irreducible flat $S$-schemes locally of finite type. Let $f: Y \rightarrow X$ be a dominant $S$-morphism. Let $s \in S$. Suppose that $f$ is quasi-finite at some point $y_{0} \in Y_{s}$, and that $Y_{s}$ is irreducible at $y_{0}$. Then $X_{s}$ is irreducible at $x_{0}:=f\left(y_{0}\right)$.

Proof. The property is local on $X$ and $Y$. In particular, shrinking $X$ and $Y$ if necessary, we can suppose that $f: Y \rightarrow X$ is quasi-finite and separated. Thus $f$ can be factorized as $Y \rightarrow \bar{Y} \rightarrow X$ with an open (dense) immersion followed by a finite surjective morphism ([5], IV.8.12.6). Let $Z_{1}, Z_{2}$ be two irreducible components of $X_{s}$ passing through $x_{0}$. By the going-down property of $\bar{Y} \rightarrow X([10], 5 . \mathrm{E} .(\mathrm{v}))$, there exist irreducible closed subschemes $F_{1}, F_{2}$ of $\bar{Y}$ passing through $y_{0}$ such that the induced maps $F_{i} \rightarrow Z_{i}(i=1,2)$ are finite and dominant (thus surjective). Let $\eta$ be the generic point of $S$. As $X, Y$ are irreducible and flat over $S$, both $X_{s}$ and $Y_{s}$ are equidimensional of dimension $\operatorname{dim} X_{\eta}=\operatorname{dim} Y_{\eta}$ ([5], IV.14.2.3). Consequently,

$$
\operatorname{dim} F_{i}=\operatorname{dim} Z_{i}=\operatorname{dim}_{x_{0}} X_{s}=\operatorname{dim} X_{\eta}=\operatorname{dim} Y_{\eta}=\operatorname{dim}_{y_{0}} Y_{s}
$$

and $F_{i} \cap Y_{s}=Y_{s}$. Therefore $Z_{1}=Z_{2}$ and $X_{s}$ is irreducible at $x_{0}$. 
Lemma 3.4. Let $S$ be a locally noetherian scheme, let $X, Y$ be two $S$-schemes locally of finite type, and let $f: Y \rightarrow X$ be a morphism of $S$-schemes. Consider $s \in S$, and $y_{0} \in Y_{s}$ a closed point of $Y_{s}$. Let $x_{0}=f\left(y_{0}\right)$. Suppose that $Y_{s}$ is regular at $y_{0}$ and

$$
\operatorname{dim}_{y_{0}} Y_{s}>1 \text { and } \operatorname{codim}_{y_{0}}\left(f^{-1}\left(x_{0}\right), Y_{s}\right)>0
$$

(The second inequality means that $f$ is non-constant on the irreducible component of $Y_{s}$ containing $\left.y_{0}\right)$. Then there exists a subscheme $Z$ of $Y$ passing through $y_{0}$ such that

$$
\operatorname{dim}_{y_{0}} Z_{s}<\operatorname{dim}_{y_{0}} Y_{s} \text { and } \operatorname{codim}_{y_{0}}\left(f^{-1}\left(x_{0}\right) \cap Z, Z_{s}\right)>0,
$$

and $Z_{s}$ is regular at $y_{0}$. If furthermore $Y$ is regular (resp. if $Y \rightarrow S$ is flat) at $y_{0}$, we can assume that the same property holds for $Z$.

Proof. We construct $Z$ locally at $y_{0}$ as a hypersurface defined by some $u \in \mathfrak{m}_{y_{0}} \mathcal{O}_{Y, y_{0}}$ which must avoid some ideals of $\mathcal{O}_{Y, y_{0}}$. We notice the following facts:

(i) Let $\Gamma_{1}, \ldots, \Gamma_{n}$ be the irreducible components of $f^{-1}\left(x_{0}\right)$ of codimension 1 in $Y_{s}$ passing through $y_{0}$. Locally at $y_{0}$, each $\Gamma_{i}$ is defined by a prime principal ideal $\bar{t}_{i} \mathcal{O}_{Y_{s}, y_{0}} \subseteq \mathfrak{m}_{y_{0}} \mathcal{O}_{Y_{s}, y_{0}}$ because $Y_{s}$ is regular at $y_{0}$. As $\operatorname{dim}_{y_{0}} Y_{s}>1$, we have $\mathfrak{m}_{y_{0}} \mathcal{O}_{Y_{s}, y_{0}} \nsubseteq \bar{t}_{i} \mathcal{O}_{Y_{s}, y_{0}}$;

(ii) We have $\mathfrak{m}_{y_{0}} \mathcal{O}_{Y_{s}, y_{0}} \nsubseteq \mathfrak{m}_{y_{0}}^{2} \mathcal{O}_{Y_{s}, y_{0}}$ because $Y_{s}$ has positive dimension at $y_{0}$.

By prime avoidance lemma ([10], 1.B), there exists

$$
\bar{u} \in \mathfrak{m}_{y_{0}} \mathcal{O}_{Y_{s}, y_{0}} \backslash\left(\mathfrak{m}_{y_{0}}^{2} \mathcal{O}_{Y_{s}, y_{0}} \cup\left(\cup_{i \leq n} \bar{t}_{i} \mathcal{O}_{Y_{s}, y_{0}}\right)\right)
$$

Lift $\bar{u}$ to some $u \in \mathfrak{m}_{y_{0}} \mathcal{O}_{Y, y_{0}}$ and let $Z:=V(u)$ be the subscheme of $Y$ defined in some open neighborhood of $y_{0}$. Then:

(1) $Z_{s}$ is regular at $y_{0}$; if $Y$ is regular (resp. if $Y \rightarrow S$ is flat) at $y_{0}$, then the same holds for $Z$ because $u \notin \mathfrak{m}_{y_{0}}^{2} \mathcal{O}_{Y, y_{0}}$ and $\bar{u}$ is not a zero divisor);

(2) $\operatorname{dim}_{y_{0}} Z_{s}<\operatorname{dim}_{y_{0}} Y_{s}$

(3) and $\operatorname{codim}_{y_{0}}\left(f^{-1}\left(x_{0}\right) \cap Z, Z_{s}\right)>0$, because otherwise $V(\bar{u})$ would be contained in, hence equal to, some irreducible component of $f^{-1}\left(x_{0}\right)$, so $\bar{u} \in \bar{t}_{i} \mathcal{O}_{Y_{s}, y_{0}}$ for some $i \leq n$. Contradiction.

Therefore $Z$ satisfies the desired properties.

Lemma 3.5. Let $S$ be a Dedekind scheme. Let $X$ be a normal relative curve over $S, 3$ and let $Y$ be a regular scheme which is flat and locally

\footnotetext{
${ }^{3}$ By a relative curve over $S$, we mean a flat, locally finite type $S$-scheme with generic fiber of dimension 1 .
} 
of finite type over $S$. Suppose $Y_{s}$ is regular. Let $f: Y \rightarrow X$ be an $S$-morphism, and $x_{0}=f\left(y_{0}\right)$ for some $y_{0} \in Y_{s}$. Suppose that

$$
\operatorname{codim}_{y_{0}}\left(f^{-1}\left(x_{0}\right), Y_{s}\right)>0 \text {. }
$$

Then $X_{s}$ is irreducible and reduced at $x_{0}$. If $Y_{s}$ is geometrically reduced in a neighborhood of $y_{0}$, then $X_{s}$ is geometrically reduced in a neighborhood of $x_{0}$.

Proof. We can suppose $X, Y$ are integral. Using repeatedly Lemma 3.4, we find a subscheme $Z$ of $Y$ containing $y_{0}$, flat over $S$, such that $Z_{s}$ is irreducible and regular of dimension 1 and $\left.f\right|_{Z_{s}}$ is non-constant. This implies that $\left.f\right|_{Z_{s}}$ is quasi-finite and $\left.f\right|_{Z}$ is dominant. By Lemma 3.3, $X_{s}$ is irreducible at $x_{0}$. Shrinking $X$ and $Y$ if necessary, we can suppose $X_{s}$ is irreducible. As $\left.f\right|_{Z_{s}}$ is non-constant, $Y_{s} \rightarrow X_{s}$, and hence $Y \rightarrow X$, are dominant. Thus $X_{s}$ is reduced at its generic point $\xi$ because the ramification index of $\mathcal{O}_{S, s} \rightarrow \mathcal{O}_{X, \xi}$ is 1 . But $X$ is normal, hence $S_{2}$, $X_{s}$ is $S_{1}$. This implies that $X_{s}$ is reduced and $Y_{s} \rightarrow X_{s}$ is schemetheoretically dominant. Then the same property holds over $\overline{k(s)}$, which implies that $X_{s}$ is geometrically reduced if $Y_{s}$ is geometrically reduced.

Proposition 3.6. Let $S$ be a Dedekind scheme, and let $X$ be a normal relative curve over $S$ with smooth generic fiber. Let $f: Y \rightarrow X$ be a morphism with $Y$ smooth and $Y_{s}$ irreducible for some closed point $s \in S$. Then either $f\left(Y_{s}\right)$ is one point, or $X$ is smooth at every point of $f\left(Y_{s}\right)$.

Proof. We may assume that $S=\operatorname{Spec}(R)$ is local, and that $f\left(Y_{s}\right)$ is not one point. Then for all $y_{0} \in Y_{s}$ and $x_{0}:=f\left(y_{0}\right)$, we have $\operatorname{codim}_{y_{0}}\left(f^{-1}\left(x_{0}\right), Y_{s}\right)>0$. Let $R^{\prime}$ be any discrete valuation ring dominating $R$. Then $X_{R^{\prime}}:=X \otimes_{R} R^{\prime}$ has smooth generic fiber, and its special fiber is reduced at any point $x_{0}^{\prime}$ lying over $x_{0}$ by Lemma 3.5. So $X_{R^{\prime}}$ is normal at $x_{0}^{\prime}$. Therefore, to prove $X$ is smooth at $x_{0}$, we can enlarge $R$ and suppose it is complete with algebraically closed residue field. Using Lemma 3.4, we can suppose that $Y$ is a relative smooth curve. Then $f$ is quasi-finite, and $\operatorname{Spec} \widehat{\mathcal{O}}_{Y, y_{0}} \rightarrow \operatorname{Spec} \widehat{\mathcal{O}}_{X, x_{0}}$ is finite. By a result of Raynaud ([13], Appendice, p. 195), $X$ is smooth at $x_{0}$.

Remark 3.7 Let $X$ be an integral relative curve over $S$ with smooth generic fiber. Then $X$ admits a minimal desingularization $X^{\prime} \rightarrow X$, made of a finite sequence of normalizations and blowing-ups of closed singular points. See e.g. [8, 8.3.50 and 9.3.32 when $X$ is proper over 
$S$. As the construction of $X^{\prime}$ is local on $X$, and the minimal desingularization is unique, the same result holds for any integral relative curve $X$ over $S$ with smooth generic fiber.

Corollary 3.8. Let $S$ be a Dedekind scheme, and let $X$ be an integral relative curve over $S$. Let $f: Y \rightarrow X$ be an $S$-morphism from a smooth $S$-scheme $Y$ to $X$. Let $s \in S$ be a closed point.

(1) If $f\left(Y_{s}\right)$ is reduced to one point $x_{0} \in X_{s}$, then $f$ factors as $Y \rightarrow$ $\widetilde{X} \rightarrow X$, where the second morphism is the blowing-up of $X$ along the reduced center $x_{0}$.

(2) Suppose that $X_{K}$ is smooth, $Y$ is irreducible and that $f_{K}: Y_{K} \rightarrow X_{K}$ is dominant. Let $X^{\prime} \rightarrow X$ be the minimal desingularization of $X$ (Remark 3.7). Then $f: Y \rightarrow X$ factors through $X^{\prime} \rightarrow X$.

Proof. (1) We have to show that $\mathfrak{m}_{x_{0}} \mathcal{O}_{Y}$ is an invertible sheaf of ideals of $\mathcal{O}_{Y}$. Let $y \in f^{-1}\left(x_{0}\right)=Y_{s}$, and let $\pi$ be a generator of $\mathfrak{m}_{s} \mathcal{O}_{S, s}$. We have

$$
\sqrt{\mathfrak{m}_{x_{0}} \mathcal{O}_{Y, y}}=\sqrt{\pi \mathcal{O}_{Y, y}}=\pi \mathcal{O}_{Y, y}
$$

(because $Y_{s}$ is reduced). As $\pi \mathcal{O}_{Y, y} \subseteq \mathfrak{m}_{x_{0}} \mathcal{O}_{Y, y}$, we find $\mathfrak{m}_{x_{0}} \mathcal{O}_{Y, y}=\pi \mathcal{O}_{Y, y}$. Therefore $\mathfrak{m}_{x_{0}} \mathcal{O}_{Y}$ is an invertible sheaf of ideals, and $Y \rightarrow X$ factors through $Y \rightarrow \widetilde{X} \rightarrow X$.

(2) As the minimal desingularization commutes with restriction to open subsets, and because the property to prove is local at $Y$, we can suppose $Y$ is quasi-compact. Then $f(Y)$ is contained in a quasicompact open subset of $X$. Therefore we can also suppose $X$ is quasicompact.

As $Y$ is normal and $f_{K}$ is dominant, $f$ factors through the normalization of $X$. Furthermore, the normalization map of $X$ is finite (see [8], 8.3.49(d)). So we can suppose $X$ is normal.

Let $F$ be the singular locus of $X$, which is a finite closed subset of $X_{s}$. Let $X_{1} \rightarrow X$ be the blowing-up along $F$ (with the reduced structure). If $F \cap f\left(Y_{s}\right)=\emptyset$, then $f$ trivially factors as $Y \rightarrow X_{1} \rightarrow X$. In general, for any $x_{0} \in F \cap f\left(Y_{s}\right)$, it follows easily from Proposition 3.6 that $f^{-1}\left(x_{0}\right)$ is a union of irreducible components of $Y_{s}$ and, by (1), $f$ factors through $X_{1} \rightarrow X$. Similarly as above, the morphism $Y \rightarrow X_{1}$ factors through the normalization map $X_{1}^{\prime} \rightarrow X_{1}$. Now we start again with $Y \rightarrow X_{1}^{\prime}$ and the process will stop at the minimal desingularization of $X$.

\section{NÉron MODELS OF PROPER SMOOTH CURVES}

Let $S$ be a Dedekind scheme with field of functions $K$. Let $X_{K}$ be a proper regular and connected curve over $K$, of positive arithmetic 
genus (i.e., $\operatorname{dim} \mathrm{H}^{1}\left(X_{K}, \mathcal{O}_{X_{K}}\right)>0$ ). When the base $S$ is excellent or $X_{K}$ is smooth over $K, X_{K}$ admits a unique minimal proper regular model $X_{\text {min }}$ over $S$ (see [2, Theorem 1.2 or [8], 8.3.45 and 9.3.21). Let $X_{\mathrm{sm}}$ denote the smooth locus of $X_{\min } / S$. The aim of this section is to prove the next theorem. See also Proposition 4.12 for a partial result in higher dimension.

Theorem 4.1. Let $S$ be a Dedekind scheme with field of functions $K$. Let $X_{K}$ be a proper regular connected curve of positive arithmetic genus over $K$. Assume either $S$ is excellent or $X_{K} / K$ is smooth. Then $X_{\mathrm{sm}}$ is the Néron model of the smooth locus $X_{K, \mathrm{sm}}$ of $X_{K}$ over $S$.

We will deduce Theorem 4.1 from the next proposition.

Proposition 4.2 (see also [1], 7.1/6). Let $P_{K}$ be a separated connected smooth $K$-scheme of finite type, and let $U_{K} \subseteq P_{K}$ be a connected smooth closed subscheme of dimension one. Assume that $P_{K}$ admits a Néron lft-model (resp. Néron model) $P$ over $S$. Then $U_{K}$ admits a Néron lft-model (resp. Néron model) over $S$.

Proof. Let $U_{0}$ denote the scheme-theoretic closure of $U_{K}$ inside $P$. Let $p: U \rightarrow U_{0}$ be the minimal desingularization of $U_{0}$ (Remark 3.7). We want to prove that the smooth locus $U_{\mathrm{sm}}$ of $U \rightarrow S$ is the Néron lft-model of $U_{K}$ over $S$. As the formation of $U_{\text {sm }}$ commutes with localization and strict henselization ([8], Proposition 9.3.28), by Proposition 2.4 we can suppose $S=\operatorname{Spec}(R)$ is strictly local (i.e., $R$ is a strictly henselian discrete valuation ring). Let $Y$ be a smooth scheme over $S$ and let $f_{K}: Y_{K} \rightarrow U_{K}$ be a morphism of $K$-schemes. We want to extend $f_{K}$ to a morphism of $S$-schemes $Y \rightarrow U_{\mathrm{sm}}$. We can suppose $Y_{s}$ is irreducible (Remark $\left.2.3(2)\right)$. If $Y_{K}(K)=\emptyset$, then $Y=Y_{K}$, and $f_{K}$ is a morphism from $Y$ to $U$.

Suppose $Y_{K}(K) \neq \emptyset$. If $f_{K}: Y_{K} \rightarrow U_{K}$ is not dominant, the image $f_{K}\left(Y_{K}\right)$ consists of a rational point $q$ of $U_{K}$. The Zariski closure $\overline{\{q\}}$ of $\{q\}$ in $U$ is contained in $U_{\mathrm{sm}}$ (Lemma 3.1) and is the image of a section $\sigma: S \rightarrow U_{\mathrm{sm}}$. Then $f_{K}$ extends to $Y \rightarrow U_{\mathrm{sm}}$ as composition of the structure morphism $Y \rightarrow S$ and the section $\sigma: S \rightarrow U_{\mathrm{sm}}$.

Now suppose $f_{K}$ is dominant. The morphism $Y_{K} \rightarrow U_{K} \rightarrow P_{K}$ extends to a dominant morphism $Y \rightarrow U_{0}$. By Corollary $3.8(2)$, the latter induces a dominant morphism $Y \rightarrow U$. Therefore $f_{K}$ extends to $Y \rightarrow U$, hence to $Y \rightarrow U_{\mathrm{sm}}$ (Proposition 3.2). This shows that $U_{\mathrm{sm}}$ is the Néron lft-model of $U_{K}$ over $S$.

If $P$ is of finite type over $S$, then $U_{0}$ and $U$ above are of finite type over $S$, thus $U_{\text {sm }}$ is of finite type. 
The next proposition is well known.

Proposition 4.3. Let $k$ be a field, and let $C$ be a projective geometrically integral curve over $k$ of arithmetic genus $\geq 1$. Let $U \subseteq C$ be the smooth locus of $C / k$. Then the canonical morphism

$$
U \rightarrow \operatorname{Pic}_{C / k}^{1}, \quad x \mapsto \mathcal{O}_{C}(x)
$$

(given by the invertible sheaf $\mathcal{O}_{C \times{ }_{k} U}(D)$, where $D$ is the graph of the inclusion $U \rightarrow C$ ) is a closed immersion.

Proof. Let $\operatorname{Div}_{C / k}$ be the scheme of effective Cartier divisors on $C$ (see [1], §8.2). Let $\operatorname{Div}_{C / k}^{1}$ be the subscheme corresponding to effective Cartier divisors of degree 1 . Then the canonical morphism $U \rightarrow \operatorname{Div}_{C / k}^{1}$, $x \mapsto x$, is an isomorphism ([6], Exercise 9.3.8).

Let $f: \operatorname{Div}_{C / k}^{1} \rightarrow \operatorname{Pic}_{C / k}^{1}$ be the restriction of the canonical morphism $\operatorname{Div}_{C / k} \rightarrow \operatorname{Pic}_{C / k}$ (corresponding to $E \mapsto \mathcal{O}_{C}(E)$ ). It will be enough to show that $f$ is a closed immersion. It is known that $f$ can be identified to $\mathbb{P}(\mathcal{F}) \rightarrow \mathrm{Pic}_{C / k}^{1}$ for some coherent sheaf $\mathcal{F}$ on $\mathrm{Pic}_{C / k}^{1}$ ([1], Proposition $8.2 / 7)$. In particular, $f$ is proper and its fibers are projective spaces. On the other hand, the map $U \rightarrow \mathrm{Pic}_{C / k}^{1}$ is injective because $p_{a}(C)>0$. So the fiber of $f$ at any $y \in \operatorname{Im}(f)$ is a projective space of dimension 0 over $k(y)$, hence isomorphic to $\operatorname{Spec}(k(y))$. This implies that $f$ is a proper (hence closed) immersion.

Proof of Theorem 4.1: As $X_{\mathrm{sm}}$ is a finite type scheme over $S$, it is enough to show $X_{\mathrm{sm}}$ is the Néron lft-model of $X_{K, \mathrm{sm}}$. By Corollary 2.5, to show our theorem we can suppose $S$ is local. Consider the closed immersion $f: X_{K, \mathrm{sm}} \rightarrow \mathrm{Pic}_{X_{K} / K}^{1}$ defined in Proposition 4.3. On the other hand, $\operatorname{Pic}_{X_{K} / K}^{1}$ is a torsor under $J_{K}:=\operatorname{Pic}_{X_{K} / K}^{0}$, and $J_{K}$ has no subgroup isomorphic to $\mathbb{G}_{a, K}$ or $\mathbb{G}_{m, K}\left([14]\right.$, Proposition 1.1). Hence $J_{K}$ has a Néron model over $S\left([1]\right.$, Theorem 10.2/1) as well as $\operatorname{Pic}_{X_{K} / K}^{1}$ ([1], Corollary 6.5/4). By Proposition 4.2, $X_{K, \text { sm }}$ has a Néron model $N$ over $S$. Embed $N$ into a proper model, and resolve the singularities without modifying the regular locus (which contains $N$ ). Then we get a proper regular model $N^{\prime}$ containing $N$ as an open subset. The identity on $X_{K}$ extends to a morphism $N^{\prime} \rightarrow X_{\min }$. By Corollary 3.2, this morphism induces a morphism $N \rightarrow X_{\mathrm{sm}}$ which is an isomorphism on the generic fiber. Therefore $X_{\mathrm{sm}}$ satisfies the Néron mapping property.

Remark 4.4 Keep the notation of Theorem 4.1.

(1) The Néron model $X_{\mathrm{sm}}$ is not necessarily faithfully flat over $S$. Indeed, if $X_{\min }$ has a multiple fiber above some point $s \in$ 
$S$, then $\left(X_{\mathrm{sm}}\right)_{s}=\emptyset$. However, the faithful flatness holds if $X_{K}(K) \neq \emptyset$.

(2) Assume $X_{K}$ is smooth over $K$ and $X_{K}(K) \neq \emptyset$, and embed $X_{K}$ into its Jacobian $J_{K}$ by using some rational point of $X_{K}$. The proof of Theorem 4.1 shows that the smooth locus of the minimal desingularization of the scheme-theoretic closure $\overline{X_{K}} \subset$ $J$ is isomorphic to $X_{\mathrm{sm}}$, where $J$ denotes the Néron model of $J_{K}$. Note that in general, even when $X_{\min }$ is semi-stable, $X_{\mathrm{sm}} \rightarrow J$ is not an immersion, see [3], Proposition 9.5.

Remark 4.5 Suppose $S=\operatorname{Spec}(R)$ is local. Let $R \rightarrow R^{\prime}$ be an extension of discrete valuation rings of index 1 (i.e., ramification index 1 and separable residue extension), let $K^{\prime}=\operatorname{Frac}\left(R^{\prime}\right)$. Then under the condition of Theorem 4.1, $X_{\mathrm{sm}} \times_{S} \operatorname{Spec}\left(R^{\prime}\right)$ is the Néron model of $X_{K^{\prime}, \mathrm{sm}}$ over $R^{\prime}$. Indeed, the formation of $X_{\mathrm{sm}}$ commutes with completion ([8], 9.3.28). Applying Proposition 2.4 (3) to the extension $\widehat{R} \rightarrow \widehat{R}^{\prime}$, we see that $X_{\mathrm{sm}} \times{ }_{S} \operatorname{Spec}\left(\widehat{R}^{\prime}\right)$ is the $\widehat{R}^{\prime}$-Néron model, hence $X_{\mathrm{sm}} \times{ }_{S} \operatorname{Spec}\left(R^{\prime}\right)$ is the Néron $R^{\prime}$-Néron model by Proposition 2.4 (1).

Remark 4.6 For any smooth connected curve $U_{K}$ over $K$, if it can be embedded as a closed subscheme into a semi-abelian $K$-variety (or more generally, into a smooth $K$-group scheme of finite type admitting a $S$ Néron lft-model), Proposition 4.2 implies immediately that $U_{K}$ admits also an $S$-Néron lft-model. For example, this works if $U_{K}$ is proper of positive genus, or if $U_{K}$ is affine such that the reduced divisor at infinity of $U_{K}$ in its regular compactification is separable of degree $>1$ over $K$. But this method does not apply for all curves: for instance the complement of a rational point in a proper smooth connected curve can not be embedded as a closed subscheme into any semi-abelian variety over $K$. In the second part of this work (\$5-\$7), we propose a different approach which works for any affine curve. Even better, our method allows a very explicit description of the Néron lft-model with the help of minimal proper regular model of the regular compactification of the affine curve.

Corollary 4.7. Let $S$ be a Dedekind scheme with field of functions $K$, and let $X_{K}$ be a connected smooth proper curve over $K$ of positive genus. Suppose that there exist a proper smooth variety $Y_{K}$ having good reduction (i.e., having a proper smooth model $Y / S$ ) and a dominant morphism $f_{K}: Y_{K} \rightarrow X_{K}$ over $K$. Then $X_{K}$ has good reduction.

Proof. Let $X_{\mathrm{sm}}$ be the Néron model of $X_{K}$ over $S$. Then $f_{K}$ extends to $f: Y \rightarrow X_{\mathrm{sm}}$. As $Y$ is proper over $S$, the image $f(Y)$ is closed in 
$X_{\mathrm{sm}}$ and is dense because it contains $X_{K}$. So $f(Y)=X_{\mathrm{sm}}$. Therefore $X_{\mathrm{sm}}$ is proper over $S$, and $X_{K}$ has good reduction.

Remark 4.8 One can give a direct proof of Corollary 4.7 if the smooth $S$-scheme $Y / S$ is assumed to be projective. Indeed, it is enough to show $X_{\mathrm{sm}}=X_{\min }$. So one can suppose $S$ is local. Using Bertini-type result, we may find a smooth closed subscheme of $Y / S$ such that its generic fiber dominates again $X_{K}$. Then we can repeat this argument to lower the relative dimension of the scheme $Y / S$ until we find a smooth relative curve over $S$ whose generic fiber still dominates $X_{K}$. Finally we only need to apply [9], Corollary 4.10 to conclude.

Remark 4.9 Corollary 4.7 does not hold in general if $g\left(X_{K}\right)=0$. Let us consider the following example. Let $R$ be a henselian discrete valuation ring with finite residue field $k$. Let $k^{\prime} / k$ be a quadratic extension and let $T^{2}+a T+b \in R[T]$ be a lifting of the minimal polynomial of a generator of $k^{\prime} / k$. Consider the scheme

$$
X=\operatorname{Proj} R[x, y, z] /\left(x^{2}+a x y+b y^{2}+\pi z^{2}\right)
$$

where $\pi$ is a uniformizing element of $R$. Let $R^{\prime}=R[T] /\left(T^{2}+a T+b\right)$. This is a finite étale extension of $R$. Let $K=\operatorname{Frac}(R), K^{\prime}=\operatorname{Frac}\left(R^{\prime}\right)$. Then $X_{K^{\prime}} \cong \mathbb{P}_{K^{\prime}}^{1}$. So $X_{K^{\prime}}$ has good reduction over $R^{\prime}$, hence over $R$ when it is viewed as a $K$-scheme (a smooth projective model over $R^{\prime}$ is also smooth projective over $R$ ).

We have a surjective $K$-morphism $X_{K^{\prime}} \rightarrow X_{K}$. However, $X_{K}$ does not have good reduction over $S$. Indeed, suppose $X_{K}$ has a proper smooth model $P \rightarrow S$. Then $P_{k}$ is a smooth conic over a finite field, hence has a rational point. As $R$ is henselian, then $X_{K}=P_{K}$ has a rational point. The latter then specializes to the (unique) rational point of $X_{k}$. But this is a singular point of $X_{k}$. As $X$ is regular, we have a contradiction by Lemma 3.1.

Corollary 4.10. Let $X_{K}$ be a proper smooth connected curve of positive genus over $K$. Suppose that for some proper smooth connected variety $Y_{K}$ over $K$, the product $X_{K} \times_{K} Y_{K}$ has good reduction over $S$. Then $X_{K}$ has good reduction over $S$.

Proof. Apply Corollary 4.7 to the projection $X_{K} \times_{K} Y_{K} \rightarrow X_{K}$.

Let $f_{K}: Y_{K} \rightarrow X_{K}$ be a finite morphism of proper smooth and connected curves over $K$. Suppose $g\left(X_{K}\right) \geq 1$. Let $Y_{\min }, X_{\min }$ be the respective minimal proper regular models of $Y_{K}, X_{K}$ over $S$. In general, $f_{K}$ does not extend to a morphism $Y_{\min } \rightarrow X_{\min }$ (9], Remark 4.5). However, Theorem 4.1 immediately implies the next corollary, answering positively a question raised by A. Pirutka. 
Corollary 4.11. Let $f_{K}: Y_{K} \rightarrow X_{K}$ be a finite morphism of proper smooth and connected curves over $K$, with $g\left(X_{K}\right) \geq 1$. Let $Y_{\mathrm{sm}}$ (resp. $\left.X_{\mathrm{sm}}\right)$ be the smooth locus of the minimal proper regular model of $Y_{K}$ (resp. $X_{K}$ ) over $S$. Then $f_{K}$ extends to a morphism $f: Y_{\mathrm{sm}} \rightarrow X_{\mathrm{sm}}$.

For the sake of completeness, let us consider the Néron lft-models of curves of genus 0 .

Proposition 4.12. Let $X_{K}$ be a smooth projective conic over $K$.

(1) If $X_{K}=\mathbb{P}_{K}^{1}$, then $X_{K}$ does not have Néron lft-model over $S$.

(2) In general, $X_{K}$ has a Néron lft-model over $S$ if and only if $S$ is semi-local and if $X_{K}\left(K_{s}^{\mathrm{sh}}\right)=\emptyset$ for any closed point $s \in S$, where $K_{s}^{\text {sh }}$ denotes the fraction field of the strict henselization of $\mathcal{O}_{S, s}$. In this case, $X_{K}$ is its own Néron model over $S$.

Proof. (1) We will argue by contradiction: assume that $\mathbb{P}_{K}^{1}$ admits a Néron lft-model $P$ over $S$. By the Néron mapping property, there exists a morphism of $S$-schemes $f: \mathbb{P}_{S}^{1} \rightarrow P$ extending the canonical identification between the generic fibers. We claim that $f$ is an isomorphism. As $\mathbb{P}_{S}^{1}$ is proper, and $P / S$ is separated, the image $f\left(\mathbb{P}_{S}^{1}\right)$ is a closed subset of $P$. Its closed fiber has dimension 1 by Chevalley's semi-continuity theorem ([5], IV.13.1.1). Therefore $\mathbb{P}_{k(s)}^{1} \rightarrow P_{s}$ is quasifinite. By Zariski's Main Theorem, $f$ is an open immersion. But $f$ is proper and $P$ is irreducible, thus $f: \mathbb{P}_{S}^{1} \rightarrow P$ is an isomorphism.

On the other hand, there are many endomorphisms of $\mathbb{P}_{K}^{1}$ that can not extend to an endomorphism of $\mathbb{P}_{S}^{1}$, hence $\mathbb{P}_{S}^{1}$ is not the Néron lftmodel of $\mathbb{P}_{K}^{1}$. Contradiction.

(2) Assume first that $X_{K}$ admits a Néron lft-model over $S$. By Proposition $2.4(2), X_{K_{s}^{\text {sh }}}$ admits a Néron lft-model over $\operatorname{Spec}\left(\mathcal{O}_{S, s}^{\text {sh }}\right)$. As a result, $X_{K_{s}^{\text {sh }}} \neq \mathbb{P}_{K_{s}^{\text {sh }}}^{1}$. In other words, $X_{K}\left(K_{s}^{\mathrm{sh}}\right)=\emptyset$ for all closed points $s \in S$. On the other hand, this condition implies that $S$ is semilocal. Conversely, assume $X_{K}\left(K_{s}^{\text {sh }}\right)=\emptyset$ for any closed point $s \in S$. As $S$ is semi-local, $X_{K}$ is of finite type over $S$ and is its own $S$-Néron model.

Higher dimension. Let $V$ be an algebraic variety over an algebraically closed field $k$. We say that $V$ contains a rational curve if $V$ has a subscheme isomorphic to an open dense subscheme of $\mathbb{P}_{k}^{1}$. It is easy to see that if $V$ does not contain any rational curve, then for any algebraically closed field extension $K / k, V_{K}$ does not contain any rational curve.

Proposition 4.13. Let $S$ be a Dedekind scheme with field of functions $K$. Let $X_{K}$ be a smooth proper algebraic variety over $K$. Suppose $X_{K}$ 
has a proper regular model $X$ over $S$ such that no geometric fiber $X_{\bar{s}}$, $s \in S$, contains a rational curve. Then the smooth locus $X_{\mathrm{sm}}$ of $X$ is the Néron model of $X_{K}$.

Proof. Let $Y$ be an irreducible smooth scheme of finite type over $S$ and let $f_{K}: Y_{K} \rightarrow X_{K}$ be a morphism of $K$-schemes. Consider the $Y$ scheme $Z:=X \times{ }_{S} Y \rightarrow Y$. Its geometric fibers are $Z_{\bar{y}}=X_{s} \otimes_{k(s)} \overline{k(y)}$ and they do not contain any rational curve. The morphism $f_{K}$ induces a section $Y_{K} \rightarrow Z_{K}, y \mapsto\left(f_{K}(y), y\right)$, which extends to a section $Y \rightarrow Z$ by [4], Proposition 6.2. Composing this section with the projection $Z \rightarrow X$ gives a morphism $f: Y \rightarrow X$ extending $f_{K}$. By Proposition 3.2, $f(Y) \subseteq X_{\mathrm{sm}}$. This proves that $X_{\mathrm{sm}}$ is the Néron model of $X_{K}$ over $S$.

Remark 4.14 If $A_{K}$ is an abelian variety having good reduction over $S$, then we recover the well-known fact that a proper smooth model $A$ of $A_{K}$ is the Néron model of $A_{K}$ ([1], Proposition 1.2/8).

\section{NÉRON LFT-MODELS OF OPEN CURVES IN THE LOCAL CASE}

Let $X_{K}$ be a separated smooth connected curve having a Néron lftmodel $X$ over $S$ (e.g. if $X_{K}$ is proper of positive genus, see Theorem 4.1). Let $U_{K}$ be an open dense subscheme of $X_{K}$. A natural question is whether $U_{K}$ has a Néron lft-model $U$ and, if it exists, how it is related to $X$. In this section, we restrict ourselves to the case where $S=\operatorname{Spec}(R)$ is local. Then we will show that the answer is positive under mild hypothesis and we describe explicitly the construction of $U$.

Let $R^{\mathrm{sh}}$ denote the strict henselization of $R, \widehat{R^{\mathrm{sh}}}$ the completion of $R^{\mathrm{sh}}, K^{\mathrm{sh}}=\operatorname{Frac}\left(R^{\mathrm{sh}}\right)$ and $\widehat{K^{\mathrm{sh}}}=\operatorname{Frac}\left(\widehat{R^{\mathrm{sh}}}\right)$.

\subsection{Main statement.}

Theorem 5.1. Let $X_{K}$ be a separated smooth connected curve over $K$ and let $U_{K}$ be a dense open subscheme of $X_{K}$. Suppose that $X_{K}$ has a smooth model $X$ over $S$ such that $X_{\widehat{R^{\text {sh }}}}$ is the Néron lft-model of $X_{\widehat{K^{\text {sh }}}}$. Then $U_{K}$ has a Néron lft-model $U$ over $S$. Moreover, denoting by $\Delta_{K}=X_{K} \backslash U_{K}$ the boundary of $U_{K}$ in $X_{K}$, U satisfies the following properties.

(1) The scheme $U$ is of finite type over $S$ if and only if $X$ is of finite type and if

$$
\Delta_{K} \cap X_{K}\left(\widehat{K^{\mathrm{sh}}}\right)=\emptyset .
$$

If $S$ is excellent, the latter condition is also equivalent to

$$
\Delta_{K} \cap X_{K}\left(K^{\text {sh }}\right)=\emptyset ;
$$


(2) Let $\Delta$ be the Zariski closure of $\Delta_{K}$ in $X$. Then the identity on the generic fiber $U_{K}$ extends to an open immersion $X \backslash \Delta \rightarrow U$, and the open immersion $U_{K} \rightarrow X_{K}$ extends to a morphism $U \rightarrow X$.

(3) Let $s$ be the closed point of $S$ and let $k(s)^{\text {sep }}$ be a separable closure of the residue field $k(s)$ of $s$. Then $U=X \backslash \Delta$ if and only if $\Delta \cap X_{s}\left(k(s)^{\mathrm{sep}}\right)=\emptyset$.

Remark 5.2 In the statement of Theorem 5.1, the $S$-model $X$ is necessarily the Néron lft-model of $X_{K}$ over $S$ (Proposition 2.4(1)), but the requirement in Theorem 5.1 is slightly stronger than this, except when $S$ is excellent (Proposition $2.4(3)$ ).

5.2. Construction of $U$. Let $k=k(s)$. First we construct a (possibly infinite) sequence of blow-ups of $X$, then define $U$ as a suitable open subset of the resulting scheme. Note that the following construction can be done for any smooth $S$-scheme $X$ such that $U_{K}$ is a dense open subset of $X_{K}$, and Lemmas 5.3 and 5.4 hold just under these assumptions.

Put $X_{0}:=X$ and $\Delta_{0}:=\Delta$.

(i) Let $\Delta_{0}^{\prime}=\left(\Delta_{0}\right)_{s} \cap \operatorname{sm}\left(X_{0}\right)_{s}\left(k^{\mathrm{sep}}\right)$, i.e., the subset of points of $\left(\Delta_{0}\right)_{s} \cap$ $\operatorname{sm}\left(X_{0}\right)_{s}$ with separable residue field over $k$. If $\Delta_{0}^{\prime}=\emptyset$, we stop.

(ii) Otherwise, let $X_{1} \rightarrow X_{0}$ be the blowing-up of $X_{0}$ along $\Delta_{0}^{\prime}$ (endowed with the reduced structure). Let $\Delta_{1}$ be the Zariski closure of $\Delta_{K}$ in $X_{1}$ and let $\Delta_{1}^{\prime}=\Delta_{1} \cap \operatorname{sm}\left(X_{1}\right)_{s}\left(k^{\mathrm{sep}}\right)$. If $\Delta_{1}^{\prime}=\emptyset$, we stop.

(iii) Otherwise blow up $X_{2} \rightarrow X_{1}$ along $\Delta_{1}^{\prime}$ (reduced) and start again with the Zariski closure $\Delta_{2}$ of $\Delta_{K}$ in $X_{2}$. We construct in this way a (possibly infinite) sequence of models locally of finite type $X_{n}$ of $X_{K}$, with $\Delta_{n} \subset X_{n}$ the Zariski closure of $\Delta_{K}$ in $X_{n}$, and $X_{n+1} \rightarrow X_{n}$ is the blowing-up of $\Delta_{n}^{\prime}:=\Delta_{n} \cap \operatorname{sm}\left(X_{n}\right)_{s}\left(k^{\text {sep }}\right)$.

Let $U_{n}=\operatorname{sm}\left(X_{n}\right) \backslash \Delta_{n}$. The identity map on $U_{K}$ extends to an open immersion $U_{n} \rightarrow U_{n+1}$. More precisely $U_{n}$ is $U_{n+1}$ minus the the exceptional divisor of $X_{n+1} \rightarrow X_{n}$. Let $U=\bigcup_{n \geq 0} U_{n}$. This is a smooth, separated scheme locally of finite type over $S$, with generic fiber isomorphic to $U_{K}$. We will show that $U$ is the Néron lft-model of $U_{K}$ over $S$. By construction, we have canonical morphisms

$$
X \backslash \Delta \hookrightarrow U \rightarrow X
$$

and the second morphism has image in $(X \backslash \Delta) \cup\left(\Delta_{0}^{\prime}\right)_{s}$.

Note that the formation of $U$ commutes with any flat extension of discrete valuation rings $R \rightarrow R^{\prime}$ because taking Zariski closure, blowing-up and taking the smooth locus are all compatible with such an extension. 
To prove $U$ is the Néron lft-model, we can replace $R$ by $\widehat{R^{\mathrm{sh}}}$ and suppose $R$ is strictly henselian and excellent (Proposition 2.4). After this reduction, $X$ is the Néron lft-model of $X_{K}$ in the situation of Theorem 5.1. But in general the curve $U_{K}$ might be no longer connected (the curve $X_{K}$ and its Néron lft-model decompose accordingly). In this situation, if we can deal with each connected component of $U_{K}$ (which is an open subscheme of a connected component of $X_{K}$ ), the general case follows (Remark $2.3(3)$ ). Hence, we may assume that $U_{K}$ is still connected.

5.3. Dilatation. ([1], §3.2). Let $R$ be a discrete valuation ring. Let $X$ be any flat $R$-scheme of finite type. Let $E$ be a closed subscheme of the special fiber $X_{s}$ defined by a sheaf of ideals $\mathcal{I} \subset \mathcal{O}_{X}$. Recall that the dilatation of $E$ on $X$ is obtained by blowing-up $u: \widetilde{X} \rightarrow X$ along $E$, and then taking the open subset of $\widetilde{X}$ where $u^{*} \mathcal{I}$ is generated by a uniformizing element of $R$. Denote by $X^{\prime}$ the dilatation of $E$. It satisfies the following universal property: for any flat $R$-scheme $Z$, a morphism $Z \rightarrow X$ factors through $X^{\prime} \rightarrow X$ if and only if $Z_{s} \rightarrow X_{s}$ factors through $E \rightarrow X_{s}$ as morphism.

If $X / R$ is smooth and if the center $E$ is smooth over $k$, by a local computation, one can show that the dilatation $X^{\prime}$ of $E$ on $X$ is smooth over $R$, and is equal to the complement in $\widetilde{X}$ of the strict transform of $X_{s}$.

\subsection{Some technical lemmas.}

Lemma 5.3. Keep the notation of $\$ 5.2$ and assume $R$ is strictly henselian and excellent. Suppose that the sequence of blowing-ups $X_{n+1} \rightarrow$ $X_{n}$ is infinite. Let $p_{1}, p_{2} \in X_{K}$ be closed points such that for all $n \in \mathbb{N}$, they specialize to a same point $x_{n} \in \Delta_{n}^{\prime}$. Then $p_{i} \in X_{K}(K)$ and $p_{1}=p_{2}$.

Proof. Let us first prove $p_{i} \in X_{K}(K)$. Let $p \in\left\{p_{1}, p_{2}\right\}$. Let $X_{n+1}^{\prime} \subseteq$ $X_{n+1} \rightarrow X_{n}$ be the dilatation of $\Delta_{n}^{\prime}$ on $X_{n}$ and let $P_{n}=\left\{p, x_{n}\right\}$ be the reduced Zariski closure of $p$ in $X_{n}$. By the construction of $X_{n}, x_{n+1}$ maps to $x_{n} \in \Delta_{n}^{\prime}$ and it is a smooth point of $X_{n+1}$. So $x_{n+1} \in X_{n+1}^{\prime}$ and for all $n \geq 0$, we have a commutative diagram

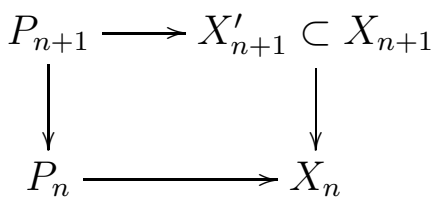

The first vertical arrow is birational and finite. Moreover, $P_{n+1}$ is nothing but the strict transform of $P_{n}$ in $X_{n+1}$. By the embedded 
resolution of singularities (see e.g., [8], 9.2.26, recall that $S$ is excellent), there exists $m \geq 1$ such that $P_{m}$ is a regular scheme. Then $P_{m+1} \rightarrow P_{m}$ is an isomorphism and we have a factorization

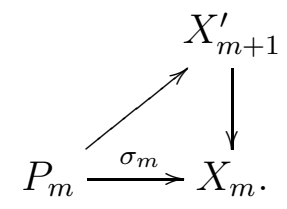

By the universal property of the dilatation (\$5.3), the closed immersion $\sigma_{m, s}: P_{m, s} \rightarrow X_{m, s}$ factors through Spec $k\left(x_{m}\right) \subset X_{m, s}$. Hence $P_{m, s} \rightarrow$ Spec $k\left(x_{m}\right)=$ Spec $k$ is an isomorphism. Therefore $P_{m} \rightarrow S$ is an isomorphism and we have $K(p)=K$.

If $p_{1} \neq p_{2}$, by the embedded resolution of singularities of the Zariski closure of $\left\{p_{1}, p_{2}\right\}$ in $X_{0}$, the Zariski closure becomes a disjoint union of sections in some $X_{n}$. Contradiction with the hypothesis in the lemma.

Lemma 5.4. Keep the notation of $\$ 5.2$ and suppose $R$ is strictly henselian and excellent.

(a) If $\left(X_{n}\right)_{n}$ is an infinite sequence, and if $\left(x_{n}\right)_{n}$ is such that $x_{n} \in \Delta_{n}^{\prime}$ and $x_{n+1} \mapsto x_{n}$ by $X_{n+1} \rightarrow X_{n}$ for all $n \geq 0$, then there exists $p \in \Delta_{K}$ such that $x_{n} \in P_{n}$ (Zariski closure of $p$ in $X_{n}$ ) for all $n \geq 0$.

(b) Let $Y$ be a flat integral $S$-scheme of finite type with irreducible closed fiber $Y_{s}$. Let $f_{K}: Y_{K} \rightarrow X_{K}$ be a morphism. Suppose that for all $n \geq 1, f_{K}$ extends to $f_{n}: Y \rightarrow X_{n}$ and that $f_{n}\left(Y_{s}\right) \subseteq \Delta_{n}^{\prime}$. Then $f_{K}\left(Y_{K}\right) \cap \Delta_{K} \neq \emptyset$.

(c) The scheme $U$ is of finite type over $S$ if and only if $X$ is of finite type and $\Delta_{K} \cap X_{K}(K)=\emptyset$.

Proof. (a) Let $F_{n} \subseteq \Delta_{K}$ be the set of points specializing to $x_{n}$. Then $\left(F_{n}\right)_{n}$ is a decreasing sequence of non-empty finite sets, so $\cap_{n} F_{n} \neq \emptyset$ and any point $p$ in the intersection satisfies the required property.

(b) As $Y_{s}$ is irreducible, $f_{n}\left(Y_{s}\right)$ is reduced to one point $x_{n}$ and $x_{n+1} \mapsto$ $x_{n}$ by $X_{n+1} \rightarrow X_{n}$. Let $p \in \Delta_{K}$ be such that $x_{n} \in P_{n}$ for all $n \geq 0$. Let $q \in Y_{K}$ be a lifting of some closed point of $Y_{s}$. Then $f_{K}(q)$ and $p$ are closed points of $X_{K}$ having the same specialization $x_{n} \in \Delta_{n}^{\prime}$ for all $n$. By Lemma 5.3, $p=f_{K}(q) \in \Delta_{K} \cap f_{K}\left(Y_{K}\right)$.

(c) Suppose there exists $p \in \Delta_{K} \cap X_{K}(K)$. For any $n \geq 0$ such that $X_{n}$ is constructed, $p$ specializes to a point of $\Delta_{n} \cap \operatorname{sm}\left(X_{n}\right)_{s}(k)$, so $X_{n+1}$ exists in the construction of $\S$ 5.2. As $X_{n+1} \rightarrow X_{n}$ consists in blowing-up some smooth points, $U_{n+1}$ contains a dense open subset of the exceptional locus of $X_{n+1} \rightarrow X_{n}$. So $U_{n} \subsetneq U_{n+1}$ and $U$ is not of 
finite type. On the other hand, if $X$ is not of finite type, then $U_{1}$, thus $U$, is not of finite type.

Conversely, if $\Delta_{K}$ does not contain rational point of $X_{K}$, there exists $m \geq 1$ such that no point of $\Delta_{K}$ specializes to a point of $\operatorname{sm}\left(X_{m}\right)_{s}(k)$ (Lemma 5.3) , and the construction of $\S 5.2$ stops at this step. Thus $U=U_{m}$ is of finite type over $S$ if $X$ is of finite type.

Lemma 5.5. Let $S$ be a locally noetherian scheme, and let $f: Z \rightarrow$ $T$ be a morphism of locally noetherian flat $S$-schemes. Let $z_{0} \in Z_{s}$ and $t_{0}=f\left(z_{0}\right)$. Suppose $Z_{s} \rightarrow T_{s}$ is flat at $z_{0}$. Then the canonical morphism

$$
f_{z_{0}}: \operatorname{Spec} \mathcal{O}_{Z, z_{0}} \rightarrow \operatorname{Spec} \mathcal{O}_{T, t_{0}}
$$

is surjective. In particular, for any $P \in T$ such that $t_{0} \in \overline{\{P\}}$, there exists $Q \in f^{-1}(P)$ such that $z_{0} \in \overline{\{Q\}}$.

Proof. By the fiberwise flatness criterion ([5], IV.11.3.10.1), $f$ is flat at $z_{0}$ and $f_{z_{0}}$ is a flat morphism of local schemes, hence faithfully flat. In particular, $f_{z_{0}}$ is surjective. The last assertion results from the usual interpretation of the images of $\operatorname{Spec} \mathcal{O}_{T, t_{0}}$ and $\operatorname{Spec} \mathcal{O}_{Z, z_{0}}$ in $T$ and $Z$ respectively.

5.5. Proof of Theorem $\mathbf{5 . 1}$. Now we prove that $U$ is the Néron lftmodel of $U_{K}$ over $S$. As noticed previously in \$5.2, we can suppose $S$ is strictly local and excellent. In particular $k^{\mathrm{sep}}=k$.

Let $Y$ be a smooth scheme over $S$ and let $f_{K}: Y_{K} \rightarrow U_{K}$ be a morphism of $K$-schemes. We want to extend $f_{K}$ to a morphism $Y \rightarrow U$. We can suppose $Y_{s}$ is irreducible (Remark 2.3). First $f_{K}$ extends to a morphism $f_{0}: Y \rightarrow X$ by the hypothesis on $X$. Consider the sequence $\left(X_{n}\right)_{n}$ constructed in $\$ 5.2$.

(A) Suppose that for some $m \geq 0, f$ factors through $f_{m}: Y \rightarrow X_{m}$ and that $f_{m}\left(Y_{s}\right) \nsubseteq \Delta_{m}^{\prime}$. Let us show $f_{m}(Y) \subseteq U_{m}$ or, equivalently, that $f_{m}\left(Y_{s}\right) \cap \Delta_{m}=\emptyset$ because $f_{m}(Y) \subseteq \operatorname{sm}\left(X_{m}\right)$. If this is true, then $f_{K}$ extends to $f_{m}: Y \rightarrow U_{m} \subseteq U$ and we are done. We distinguish two cases:

Case 1: $f_{m}\left(Y_{s}\right)=\left\{x_{m}\right\}$ is a singleton. By hypothesis, $x_{m} \notin \Delta_{m}^{\prime}$. As $Y_{s}$ is smooth and $Y_{s} \rightarrow \operatorname{Spec} k$ factors through $Y_{s} \rightarrow \operatorname{Spec} k\left(x_{m}\right)$, we have $x_{m} \in \operatorname{sm}\left(X_{m}\right)_{s}(k)$. So $x_{m} \notin \Delta_{m}$ and $f_{m}\left(Y_{s}\right) \subseteq U_{m}$.

Case 2: $f_{m}\left(Y_{s}\right)$ is not a singleton. If there exists $x_{m} \in f_{m}\left(Y_{s}\right) \cap \Delta_{m}$, let $y_{m} \in f_{m}^{-1}\left(x_{m}\right)$ and let $p \in \Delta_{K}$ specializing to $x_{m}$. As $\operatorname{sm}\left(X_{m}\right)_{s}$ is a smooth curve and $\left(f_{m}\right)_{s}$ is dominant, $\left(f_{m}\right)_{s}$ is flat at $y_{m}$. By Lemma 5.5 applied to $Z=Y$ and $T=\operatorname{sm}\left(X_{m}\right)$, we find a closed point $q \in Y_{K}$ such that $f_{K}(q)=p$. This contradicts the hypothesis that $f_{K}\left(Y_{K}\right) \subseteq U_{K}$. 
(B) Now we show that the condition in (A) is satisfied for some $m \geq 0$. We start with $f_{0}: Y \rightarrow X_{0}$. If $f_{0}\left(Y_{s}\right) \subseteq \Delta_{0}^{\prime}$, then $f_{0}\left(Y_{s}\right)=\left\{x_{0}\right\}$. As in Corollary 3.8(1), $f_{0}$ factors through $f_{1}: Y \rightarrow X_{1}$. If $f_{1}\left(Y_{s}\right) \nsubseteq \Delta_{1}^{\prime}$, we are done. Otherwise, we have a $f_{2}: Y \rightarrow X_{2}$. Repeating this construction, we see that if $(\mathrm{A})$ is never satisfied, then for all $n \geq 0$, $f_{K}$ extends to $f_{n}: Y \rightarrow X_{n}$ with $f_{n}\left(Y_{s}\right) \subseteq \Delta_{n}^{\prime}$. This is impossible by Lemma 5.4(b). Hence $U$ is the Néron lft-model of $U_{K}$.

It remains to prove the various properties of $U$. We first remark that if $S$ is excellent, then $K^{\text {sh }}$ is algebraically closed in its completion $\widehat{K^{\mathrm{sh}}}$, hence the two conditions in Part (1) are indeed equivalent. Part (1) is a direct consequence of Lemma 5.4(C). Parts (2) and (3) follow from the construction in 5.2: $X \backslash \Delta_{0}=U_{0}$ is open in $U$ and its generic fiber is $X_{K} \backslash \Delta_{K}=U_{K}$; and $U=X \backslash \Delta_{0}$ is equivalent to $\Delta_{0}^{\prime}=\emptyset$.

As an application of Theorem 5.1, we have the following result which will be used in Proposition 6.1 in the next section.

Proposition 5.6. Let $S$ be local. Let $P$ be a regular, proper semi-stable model of $\mathbb{P}_{K}^{1}$ over $S$. Let $\Gamma_{1}, \cdots, \Gamma_{n}$ be disjoint sections, $n \geq 2$, in the smooth locus $\operatorname{sm}(P / S)$ such that $\Gamma:=\cup_{i} \Gamma_{i}$ meets every exceptional divisor of $P / S$ when $P_{s}$ is not irreducible. Let $V_{K}=\mathbb{P}_{K}^{1} \backslash \Gamma_{K}$. Let $V$ be the $S$-model over $S$ obtained by the process described in \$5.2 starting with $X:=\operatorname{sm}(P / S)$. Then $V$ is the $S$-Néron lft-model of $V_{K}$ over $S$. Moreover, the identity $V_{K} \rightarrow V_{K}$ and the inclusion $V_{K} \rightarrow P_{K}$ extend to

$$
\operatorname{sm}(P / S) \backslash \Gamma \rightarrow V \rightarrow \operatorname{sm}(P / S)
$$

and the first morphism is an open immersion.

Proof. As the formation of $V$ commutes with completion and strict henselization, by Proposition 2.4 (1), we can suppose $S$ is strictly local and excellent. We prove the result by induction on the number of irreducible components of $P_{s}$.

First suppose $P_{s}$ is irreducible. Start with the case $n=2$. Then $P \cong \mathbb{P}_{S}^{1}$. One can see easily that $V$, which is isomorphic to the Néron lft-model of $\mathbb{G}_{m, K}$, is obtained by the process described in $\$ 5.2$ with $X:=P$. See [1], 10.1 .

If $P_{s}$ is irreducible and $n \geq 3$, we consider $U_{K}=\mathbb{P}_{K} \backslash\left\{\left(\Gamma_{1}\right)_{K},\left(\Gamma_{2}\right)_{K}\right\}$ with its Néron lft-model $U$. Then $V$ can be obtained by the process of $\$ 5.2$ starting with $X:=U$. As $P \backslash\left(\Gamma_{1} \cup \Gamma_{2}\right)$ is open in $U$ by the above discussions, and the Zariski closure of $\left(\Gamma_{i}\right)_{K}$ in $P$ is $\Gamma_{i} \subset P \backslash\left(\Gamma_{1} \cup \Gamma_{2}\right)$ when $i \geq 3$, Theorem 5.1 says that $V$ is the $S$-Néron lft-model of $V_{K}$, $P \backslash \Gamma$ is open in $V$ and the latter maps to $U$, hence to $P$.

Now suppose $P_{s}$ has more than one component. Let $E$ be an exceptional divisor in $P$. Up to renumbering, we can suppose $\Gamma_{1}, \ldots, \Gamma_{r}$, 
$r \leq n-1$, are exactly the sections of $P$ among the $\Gamma_{i}$ 's not meeting $E$. Let $\pi: P \rightarrow Q$ be the contraction of $E$ et let $q=\pi(E) \in Q_{s}$. Consider $U_{K}=\mathbb{P}_{K}^{1} \backslash\left\{\left(\Gamma_{1}\right)_{K}, \ldots,\left(\Gamma_{r}\right)_{K},\left(\Gamma_{r+1}\right)_{K}\right\}$. Then $Q$ is regular, proper and semi-stable and, if we still denote by $\Gamma_{i}$ the Zariski closure of $\left(\Gamma_{i}\right)_{K}$ in $Q$, $\Gamma_{1}, \ldots, \Gamma_{r}, \Gamma_{r+1}$ correspond to $r+1$ disjoint sections of $Q / S$ whose union meets every exceptional divisor of $Q / S$ when $Q_{s}$ is not irreducible. By the induction hypothesis, the Néron lft-model $U$ of $U_{K}$ is obtained by the process of $\$ 5.2$ starting from $X:=\operatorname{sm}(Q / S)$. As $\left(\Gamma_{r+1}\right)_{s}=\{q\}$ and $\pi: P \rightarrow Q$ is the blow-up of $Q$ along $\{q\}$, by the explicit construction of $U, \operatorname{sm}(P) \backslash\left(\cup_{i \leq r+1} \Gamma_{i}\right)$ is open in $U$. For any $i \geq r+2$, the point of $\left(\Gamma_{i}\right)_{K}$ specializes to a point of $\left(\operatorname{sm}(P) \backslash\left(\cup_{i \leq r+1} \Gamma_{i}\right)\right)_{s}$. Then Theorem5.1 tells us that $V_{K}$ admits an $S$-Néron lft-model $V$, and the latter is obtained from $U$ by blowing-up the closed points $\cup_{i \geq r+2}\left(\Gamma_{i}\right)_{s}$ contained in the open subset $\operatorname{sm}(P) \backslash\left(\cup_{i \leq r+1} \Gamma_{i}\right) \subset U$, taking the smooth locus and start again etc. In particular $\operatorname{sm}(P / S) \backslash \Gamma$ is an open subscheme of $V$ and the latter maps to $\operatorname{sm}(P / S)$.

\section{NÉRON MODELS OF OPEN SUBSETS OF A SMOOTH CONIC}

In this section, we suppose $S=\operatorname{Spec}(R)$ is local and excellent. We prove the existence of the Néron model for affine open subsets of a smooth projective conic, whose complement is non-empty and consists of ramified points.

Proposition 6.1. Let $S$ be an excellent local Dedekind scheme with field of functions $K$. Let $C_{K}$ be a projective smooth conic over $K$. Let $\Delta_{K}$ be a non-empty finite closed subset of $C_{K}$ (endowed with the reduced structure) such that $\Delta_{K}\left(K^{\mathrm{sh}}\right)=\emptyset$. Then $U_{K}:=C_{K} \backslash \Delta_{K}$ admits a Néron model over $S$.

Proof. Assume first $C_{K}(K) \neq \emptyset$, or equivalently, $C_{K} \cong \mathbb{P}_{K}^{1}$. Consider a smooth proper model isomorphic to $\mathbb{P}_{S}^{1}$ of $\mathbb{P}_{K}^{1}$. After finitely blowingups along smooth separable points of the special fiber of the latter, the construction of $\$ 5.2$ gives us a regular proper semi-stable model $P$ of $C_{K} \cong \mathbb{P}_{K}^{1}$ such that the intersection $\Delta \cap \operatorname{sm}(P / S)_{s}\left(k(s)^{\text {sep }}\right)$ is empty. By successively blowing-down exceptional divisors of $P$ which do not meet the Zariski closure $\Delta$ of $\Delta_{K}$, we can suppose that:

(1) $\Delta$ meets every exceptional divisor of $P / S$ if $P_{s}$ is not irreducible and

(2) $\Delta$ meets $P_{s}$ only at singular points or smooth inseparable points.

Claim: under the above conditions, $U:=\operatorname{sm}(P / S) \backslash \Delta$ is the Néron model of $U_{K}$. 
To prove the claim we can suppose $S$ is strictly local. In particular, each (reduced) irreducible component of $P_{s}$ is isomorphic to $\mathbb{P}_{k}^{1}$. Let $Y$ be a smooth $S$-scheme with connected fibers and let $f_{K}: Y_{K} \rightarrow U_{K}$ be a morphism of $K$-schemes. Let $\Gamma_{1}, \ldots, \Gamma_{n} \subset U(S)$ be disjoint sections such that $n \geq 2, \Gamma:=\cup_{i} \Gamma_{i}$ is ample in $P$ and such that $f_{K}\left(Y_{K}\right) \not \subset \Gamma_{K}$.

Set $H=\overline{f_{K}^{-1}\left(\Gamma_{K}\right)} \subset Y$. This is a closed subset of $Y$, empty or of codimension 1. Let $Y^{\prime}:=Y \backslash H$. We claim that the restriction $\left.f_{K}\right|_{Y_{K}^{\prime}}: Y_{K}^{\prime} \rightarrow U_{K} \backslash \Gamma_{K}$ extends to a morphism $Y^{\prime} \rightarrow U$. Indeed, let $V$ be the Néron lft-model of $V_{K}:=P_{K} \backslash \Gamma_{K}$. Then $f_{K}$ induces a morphism $f^{\prime}: Y^{\prime} \rightarrow V$. By Proposition [5.6, we have a morphism $V \rightarrow \operatorname{sm}(P / S) \backslash \Gamma=U$. Consequently, $f^{\prime}$ extends to a morphism $f^{\prime \prime}: Y^{\prime \prime} \rightarrow U$ where $Y^{\prime \prime}=Y^{\prime} \cup Y_{K}$. As $Y_{s}$ is connected, the image $f_{s}^{\prime \prime}: Y_{s}^{\prime \prime} \rightarrow U_{s}$ is contained in some connected component. Let $U^{\prime}$ denote the union of the latter connected component of $U_{s}$ with $U_{K}$. Then $f^{\prime \prime}$ factors through $U^{\prime} \subset U$.

On the other hand, the scheme $U^{\prime}$ can be obtained by first blowingdown successively all the irreducible components of $P_{s}$ other than the one containing $U_{s}^{\prime}$, then removing from the resulting proper smooth model of $C_{K} \cong \mathbb{P}_{K}^{1}$ the closure of $\Delta_{K}$. In particular, the scheme $U^{\prime}$ is affine. Thus $f^{\prime \prime}$ extends to a morphism $Y \rightarrow U^{\prime} \subset U$ since $Y \backslash Y^{\prime \prime}$ is a closed subset of codimension $\geq 2$ of the normal scheme $Y$ ([1], 4.4/2). Therefore $U$ satisfies Néron mapping property, as desired.

For the general case, if $X_{K}\left(K^{\mathrm{sh}}\right)=\emptyset$, then $X_{K}$ is the $S$-Néron model of itself. Otherwise, there exists a finite unramified extension $K^{\prime}$ of $K$ such that $C_{K}\left(K^{\prime}\right) \neq \emptyset$. Since $K^{\prime} / K$ is unramified, the complement of $U_{K^{\prime}}$ in its smooth compactification $C_{K^{\prime}}$ consists of closed points which are still ramified of degree $>1$ over $K^{\prime}$. Therefore, $U_{K^{\prime}}$ admits a Néron model $U^{\prime}$ over the semi-local ring $S^{\prime}$, the normalization of $S$ in $K^{\prime}$. Hence $U_{K}$ admits also a $S$-Néron model by Proposition 7.4 and Proposition 7.5 .

Remark 6.2 Keep the notation of Proposition 6.1, and let $U$ be the $S$ Néron model of $U_{K}$. Assume $C_{K} \cong \mathbb{P}_{K}^{1}$ with a proper smooth $S$-model $C$ such that the Zariski closure $\Delta$ of $\Delta_{K}$ (with the reduced structure) is regular. Then the canonical morphism obtained from Néron mapping property $C \backslash \Delta \rightarrow U$ is an open immersion. Indeed, as $\Delta$ is regular, after blowing up all separable closed points of $\Delta$, we obtain a proper semi-stable model $P$ of $C_{K}$ such that the conditions (1) (2) in the proof of Proposition 6.1 are satisfied. By the claim in the same proof, $U=\operatorname{sm}(P / S) \backslash \overline{\Delta_{K}}$. Thereby $C \backslash \Delta$ is an open subset of $U$.

Remark 6.3 In Proposition 6.1, we can not drop the assumption that $S$ is excellent. For example, let $k$ be a field of characteristic 2, and 
$K:=k\left(t, u^{2}\right) \subset k[[t]] \subset k((t))$ with $u \in k[[t]]$ transcendental over $k(t)$. The discrete valuation on $k((t))$ induces a discrete valuation on $K$, and let $R$ be the corresponding (discrete) valuation ring (with $t \in R$ a uniformizer). The completion $\widehat{R}$ of $R$ is $k[[t]] \subset k((t))$, hence $\widehat{K}=$ $\operatorname{Frac}(\widehat{R})=k((t))$. Note that $u \in \widehat{K}$ is purely inseparable of degree 2 over $K$ (in particular, $R$ is not excellent). Consider $v=u^{2} \in K$ and let $U_{K}=\operatorname{Spec}\left(K\left[X_{1}, X_{2}\right] /\left(X_{1}^{2}-v X_{0}^{2}-X_{0}\right)\right.$. This is the underlying scheme of a unipotent group. As $v \notin K^{2}, U_{K} \neq \mathbb{A}_{K}^{1}$. On the other hand, $U_{\widehat{K}} \cong \mathbb{A}_{\widehat{K}}$. It follows that $U_{\widehat{K}} \cong \mathbb{G}_{a, \widehat{K}}$. Therefore, by [1], 10.2/2, $U_{K}$ does not admit Néron lft-model over $S$.

\section{NÉRON LFT-MODELS OF AFFine CURVES}

The aim of this section is to prove the existence of Néron lft-models of affine curves over $K$ different from the affine line (Theorem [7.10). Let $S$ be a Dedekind scheme with field of functions $K$.

\subsection{Globalizing local Néron lft-models.}

Lemma 7.1. Let $S$ be a Dedekind scheme with $K$ its field of functions. Let $U_{K}$ be a separated connected smooth curve over $K$. Suppose that

(i) for any closed point $s \in S, U_{K}$ admits a Néron lft-model $U(s)$ over $\mathcal{O}_{S, s}$;

(ii) there exists a model of finite type $U^{0}$ of $U_{K}$ over $S$ such that for all $s \in S$, the isomorphism $U_{K}^{0} \rightarrow U(s)_{K}$ extends to an open immersion $U^{0} \times{ }_{S} \operatorname{Spec}\left(\mathcal{O}_{S, s}\right) \rightarrow U(s)$.

Then $U_{K}$ admits a Néron lft-model over $S$.

Proof. The proof is inspired from that of [1, 10.1/7. The Néron lftmodel of $U_{K}$ over $S$ will be obtained by gluing the local Néron lftmodels $U(s)$ for all closed points $s \in S$. We will first extend $U(s)$ to a model $V(s)$ over $S$ which coincides with $U^{0}$ above $S \backslash\{s\}$ and then glue the various $V(s)$ in a natural way to obtain the Néron lft-model $U$ of $U_{K}$ over $S$.

Fix a closed point $s$. As $U(s)$ is locally of finite type, any connected component $U(s)_{s, \alpha}$ of $U(s)_{s}$ is open in the closed fiber $U(s)_{s}$, hence its union with $U_{K}$ is a quasi-compact open subset $U_{\alpha}(s)$ of $U(s)$. We can extend $U_{\alpha}(s)$ to a separated scheme of finite type $U_{\alpha}$ over $S$ (use [5], IV.8.10.5). As $U_{\alpha}$ and $U^{0}$ are both of finite type over $S$ and have the same generic fiber, they are $S$-isomorphic over a dense open subset $S_{\alpha} \subseteq S \backslash\{s\}$ (and the $S$-isomorphism is unique once the isomorphisms $U_{K}^{0} \rightarrow U_{K},\left(U_{\alpha}\right)_{K} \rightarrow U_{K}$ are fixed because $U_{\alpha}$ is separated over $\left.S\right)$. 
Now we glue the separated morphisms of finite type

$$
U_{\alpha} \times{ }_{S}\left(S_{\alpha} \cup\{s\}\right) \rightarrow S_{\alpha} \cup\{s\}, \quad U^{0} \times{ }_{S}(S \backslash\{s\}) \rightarrow S \backslash\{s\}
$$

above $\left(S_{\alpha} \cup\{s\}\right) \cap(S \backslash\{s\})=S_{\alpha}$. The resulting $S$-scheme $V_{\alpha}$ is separated and of finite type because these properties are satisfied above $S_{\alpha} \cup\{s\}$ and $S \backslash\{s\}$. By construction, we have canonically

$$
V_{\alpha} \times{ }_{S}(S \backslash\{s\})=U^{0} \times{ }_{S}(S \backslash\{s\}), \quad V_{\alpha} \times{ }_{S} \operatorname{Spec}\left(\mathcal{O}_{S, s}\right)=U_{\alpha}(s) .
$$

Next we glue the various $V_{\alpha}$ (when $U_{\alpha}(s)$ runs through the connected components of $U(s)_{s}$ ) with the condition $V_{\alpha} \cap V_{\alpha^{\prime}}=U^{0}$ if $\alpha \neq \alpha^{\prime}$. The resulting $S$-scheme $V(s)$ satisfies canonically

$$
V(s) \times{ }_{S}(S \backslash\{s\})=U^{0} \times{ }_{S}(S \backslash\{s\}), \quad V(s) \times{ }_{S} \operatorname{Spec}\left(\mathcal{O}_{S, s}\right)=U(s) .
$$

Hence $V(s)$ is separated and locally of finite type over $S$. Moreover, Condition (ii) implies that the isomorphism $U_{K}^{0} \rightarrow V(s)_{K}=U(s)_{K}$ extends to an open immersion $U^{0} \rightarrow V(s)$.

Finally, we glue the various $V(s)$ when $s$ runs through the closed points of $S$ with the condition $V(s) \cap V\left(s^{\prime}\right)=U^{0}$ if $s \neq s^{\prime}$. The resulting $S$-scheme $U$ is locally of finite type and $U \times{ }_{S} \operatorname{Spec}\left(\mathcal{O}_{S, s}\right) \cong U(s)$ for all $s \in S$. By Corollary 2.5, $U$ is the Néron lft-model of $U_{K}$ over $S$, as required.

Lemma 7.2. Let $S$ be a Dedekind scheme. Let $X_{K}$ be a smooth connected separated curve over $K$ and let $U_{K}$ be an open dense subscheme of $X_{K}$. Suppose that $X_{K}$ has a smooth model $X$ over $S$ such that for all closed points $s \in S, X \times{ }_{S} \operatorname{Spec}\left(\mathcal{O}_{S, s}\right)$ satisfies the property in Theorem 5.1. Then $U_{K}$ admits a Néron lft-model over $S$.

Proof. Let $\Delta_{K}:=X_{K} \backslash U_{K}, X^{0} \subset X$ any quasi-compact open subset containing $X_{K}$, and $\Delta^{0}:=\overline{\Delta_{K}} \subset X^{0}$. By Theorem 5.1, the Néron model of $U_{K}$ over $\operatorname{Spec}\left(\mathcal{O}_{S, s}\right)$ exists for all closed points $s \in S$, and by Theorem $5.1(2), U^{0}:=X^{0} \backslash \Delta^{0}$ verifies the hypothesis of Lemma 7.1. Thus we can apply Lemma 7.1 to conclude.

Proposition 7.3. Let $S$ be a Dedekind scheme. Let $X_{K}$ be a connected regular proper curve over $K$ of arithmetic genus $\geq 1$. Let $U_{K}$ be a dense open subscheme of $X_{K}$ contained in the smooth locus of $X_{K} / K$. Suppose either $S$ is excellent or the scheme $X_{K}$ is smooth over $K$. Then $U_{K}$ admits a Néron lft-model over $S$.

Proof. Let $X=X_{\mathrm{sm}}$ be the smooth locus of the minimal proper regular model of $X_{K}$ over $S$. Let $s \in S$ be a closed point. Then $X \times_{S} \widehat{\mathcal{O}_{S, s}^{\text {sh }}}$ is the Néron model of $X_{K}$ over $\widehat{\mathcal{O}_{S, s}^{\text {sh }}}$ by Theorem 4.1 and because in both 
cases the minimal proper regular as well as the its smooth locus commute with strict henselization and completion (8], 9.3.28). Applying Lemma 7.2 to $U_{K} \subseteq X_{K \text {,sm }}$, we see that $U_{K}$ has a Néron lft-model $U$ over $S$.

\subsection{Weil restriction.}

Proposition 7.4 (see also 11, 10.1/4). Let $S$ be a Dedekind scheme with field of functions $K$ and let $X_{K}$ be a separated smooth connected curve over $K$. Let $K^{\prime} / K$ be a finite extension, and let $S^{\prime}$ be the normalization of $S$ in $K^{\prime}$. Assume that

(i) $S^{\prime} \rightarrow S$ is finite (e.g., if $S$ is excellent or $K^{\prime} / K$ is separable);

(ii) $X_{K^{\prime}}$ admits a Néron lft-model (resp. Néron model) $X^{\prime}$ over $S^{\prime}$;

(iii) any quasi-compact open subset of $X^{\prime}$ is quasi-projective over $S^{\prime}$.

Then $X_{K}$ admits also a Néron lft-model (resp. Néron model) over $S$.

Proof. Let $s \in S$. Then any finite subset $F$ of $X_{s}^{\prime}:=X^{\prime} \times{ }_{S} \operatorname{Spec} k(s)$ is contained in an affine open subset of $X^{\prime}$. Indeed, $F$ is contained in a quasi-compact open subset $W$ of $X^{\prime}$. Let $V$ be an affine open neighborhood of $s$. As $S^{\prime} \times{ }_{S} V$ is finite over $V, W \times{ }_{S} V=W \times \times_{S^{\prime}}$ $\left(S^{\prime} \times{ }_{S} V\right)$ is quasi-projective over $V$, hence $F$ is contained in an affine open subset of $W \times_{S} V \subseteq X^{\prime}$.

The morphism $S^{\prime} \rightarrow S$ is finite and locally free, hence the above property implies that the Weil restriction functor $Y:=\operatorname{Res}_{S^{\prime} /{ }_{S}} X^{\prime}$ is representable by a smooth $S$-scheme locally of finite type ([1], 7.6/4). Furthermore, by the functoriality of the Weil restriction, one checks easily that $Y$ is the $S$-Néron lft-model of its generic fiber $Y_{K} \cong \operatorname{Res}_{K^{\prime} / K}\left(X_{K^{\prime}}\right)$. Finally, remark that as $X_{K} / K$ is separated, the adjunction map $X_{K} \rightarrow$ $\operatorname{Res}_{K^{\prime} / K}\left(X_{K^{\prime}}\right)=Y_{K}$ is a closed immersion, hence it suffices to apply Proposition 4.2 to conclude the existence of Néron lft-model or Néron model of $X_{K}$.

The following result is useful when we want to check the condition (iii) of Proposition 7.4 .

Proposition 7.5. (Quasi-projectivity) Let $S$ be a Dedekind scheme with field of functions $K$, and let $U$ be a connected regular relative curve over $S$ locally of finite type. Suppose that either $U_{K}$ has a smooth compactification or $S$ is excellent. Then any quasi-compact open subset of $U$ is quasi-projective over $S$ (in the sense of [5], II.5.3.1).

Proof. Let $U_{0}$ be a quasi-compact open subset of $U$. Let $U_{0} \subseteq U_{0}^{\prime}$ be a Nagata compactification. The hypothesis on $U_{K}$ or $S$ implies that there exists a desingularization morphism $Z \rightarrow U_{0}^{\prime}$ which is an isomorphism 
above $U_{0}$. So $U_{0}$ is isomorphic to an open subscheme of a regular proper flat $S$-scheme $Z$. It is enough to show that $Z \rightarrow S$ is projective. This is a theorem of Lichtenbaum when $S$ is affine ([7], Theorem 2.8 or [8], 8.3.16), but the proof works exactly in the same way in the general case: find a positive horizontal Weil divisor $H$ on $Z$ which meets all irreducible components of all fibers of $Z \rightarrow S$. As $Z$ is regular, $H$ is defined by an invertible sheaf $\mathcal{L}$ on $Z$. The hypothesis on $H$ implies that $\mathcal{L}$ is fiberwise ample, hence $\mathcal{L}$ is relatively ample for $Z \rightarrow S$.

Remark 7.6 If the Dedekind scheme $S$ is separated, then any quasiprojective scheme over $S$ is a subscheme of some $\mathbb{P}_{S}^{N}$. Indeed, $S$ then has an invertible ample sheaf ([15, Proposition 09NZ]), and one can conclude with [5], II.5.3.3.

7.3. Affine open subsets of a conic. In this Subsection, we discuss the existence of Néron lft-models of an affine open subscheme $U_{K}$ of a smooth projective conic $C_{K} / K$. Observe first that $\mathbb{A}_{K}^{1}$ does not admit Néron lft-model over $S([1], 10.1 / 8)$.

Proposition 7.7. Let $S$ be an excellent Dedekind scheme with field of functions $K$. Let $U_{K}$ be an affine open subscheme of a smooth projective conic $C_{K}$ over $K$. Suppose $U_{K}$ is not isomorphic to $\mathbb{A}_{K}^{1}$. Then $U_{K}$ admits a Néron lft-model $U$ over $S$.

Proof. If over an algebraic closure $\bar{K}$ of $K, C_{\bar{K}} \backslash U_{\bar{K}}$ contains at least two points, then there exists a finite extension $K^{\prime} / K$ such that $U_{K^{\prime}}$ is isomorphic to an open subscheme of $\mathbb{G}_{m, K^{\prime}}$. It follows from Proposition 7.4 that we can suppose $U_{K}$ is an open subscheme of $\mathbb{G}_{m, K}$. The latter has a Néron lft-model $G$ over $S$, locally on $S$ compatible with any index 1 extension (see the construction of [1], 10.1/5). Consequently, by Lemma [7.2, $U_{K}$ admits a Néron lft-model over $S$. For the rest of the proof, we can therefore suppose that $U_{\bar{K}}$ is $C_{\bar{K}}$ minus one point. So $\Delta_{K}:=C_{K} \backslash U_{K}$ consists of a single point $q_{\infty}$ which is purely inseparable of degree $>1$ over $K$ because $U_{K} \approx \mathbb{A}_{K}^{1}$.

As $C_{K}$ is smooth over $K$, there exists a separable extension $K^{\prime} / K$ such that $C_{K^{\prime}} \cong \mathbb{P}_{K^{\prime}}^{1}$. The point of $C_{K^{\prime}} \backslash U_{K^{\prime}}$ is still purely inseparable of degree $>1$ over $K^{\prime}$ because $K^{\prime} / K$ is separable. Using Proposition 7.4. we can reduce to the case $C_{K}=\mathbb{P}_{K}^{1}$. Let $P \cong \mathbb{P}_{S}^{1}$ be a smooth proper model of $\mathbb{P}_{K}^{1}$ over $S$, and let $\Delta=\overline{\left\{q_{\infty}\right\}} \subset P$. We know (Proposition 6.1) that for all closed points $s \in S, U_{K}$ admits a Néron model $U(s)$ over $\mathcal{O}_{S, s}$. To find a global Néron lft-model, it is enough to show that for $U^{0}:=P \backslash \Delta$, the canonical morphism

$$
U^{0} \times{ }_{S} \operatorname{Spec}\left(\mathcal{O}_{S, s}\right) \rightarrow U(s)
$$


is an open immersion for all $s$ contained in a dense open subset $V \subset S$ : the base change $U^{0} \times_{S} V$ satisfies then Condition (ii) of Lemma 7.1. As $S$ is excellent, so is $\Delta$. Thus the regular locus of $\Delta$ is open in $\Delta$. Shrinking $S$ if necessary, we can assume $\Delta$ is regular. Then for any closed point $s \in S$, the morphism (7.1) is an open immersion by Proposition 6.1 and Remark 6.2, and the proposition is proved.

Corollary 7.8. Let $S$ be an excellent Dedekind scheme of characteristic $p>0$, with $K$ its field of functions. Let $G_{K}$ be a connected smooth $K$-wound unipotent group of dimension 1 . Then $G_{K}$ admits a Néron lft-model over $S$.

Remark 7.9 Let $S$ be a Dedekind scheme with field of functions $K$. One can deduce from Proposition 4.12 and Proposition 4.2 that if $X_{K}$ is a connected separated smooth $K$-variety admitting Néron lft-model over $S$, then $X_{K}$ does not contain any closed subscheme isomorphic to $\mathbb{P}_{K}^{1}$ or $\mathbb{A}_{K}^{1}$. Conversely, if $X_{K}$ is the underlying scheme of a smooth commutative algebraic group over $K$, the latter condition is also sufficient for the existence of Néron lft-model when $S$ is local and excellent ([1], 10.2/2).

When $S$ is global and excellent, whether this latter condition is sufficient is still an open question. It is conjectured ([1], 10.3, Conjecture I) that the answer is yes. Some positive examples are known in [1, Chap. 10. Corollary 7.8 provides some evidence in favor of this conjecture. Together with the well-known results for abelian varieties and for tori, we deduce that when $S$ is excellent, any smooth connected $K$-algebraic group $G_{K}$ of dimension one admits a Néron lft-model over $S$ if and only if $G_{K}$ is not isomorphic to $\mathbb{G}_{a, K}$. In other words, Conjecture I of [1], 10.3 holds when $\operatorname{dim}\left(G_{K}\right)=1$. When the unipotent group scheme $G_{K}$ in Corollary 7.8 admits a regular compactification of genus $\geq 1$, or equivalently when $\operatorname{uni}\left(G_{K}\right)=0$, Corollary 7.8 is a special case of [1], $10.3 / 5$. So the new case provided here is when $\operatorname{uni}\left(G_{K}\right)>0$, or equivalently when $G_{K}$ admits a smooth compactification of genus 0 . The latter happens only when $\operatorname{char}(K)=2$ (see last paragraph of [1], 10.3, p. 316). In this case, $G_{K}$ is the subgroup of $\mathbb{G}_{a, K}^{2}=\operatorname{Spec}(K[X, Y])$ defined by the equation $X^{2}=Y+a Y^{2}$ for some $a \in K \backslash K^{2}$, which, as a scheme, is isomorphic to $\operatorname{Proj}\left(K\left[T, T^{\prime}\right]\right) \backslash V_{+}\left(T^{2}-a T^{\prime 2}\right)$.

7.4. Néron lft-models for affine curves. We are now in the position to prove the existence of Néron lft-models for affine curves.

Theorem 7.10. Let $S$ be an excellent Dedekind scheme with field of functions $K$. Let $U_{K}$ be an affine smooth connected curve over $K$. 
Then $U_{K}$ admits a Néron lft-model over $S$ if $U_{K} ¥ \mathbb{A}_{L}^{1}$ for any finite extension $L / K$.

Proof. By Lemma 2.6, we can suppose $U_{K}$ is geometrically connected. If the regular compactification of $U$ has positive arithmetic genus, then $U_{K}$ has a Néron lft-model by Proposition [7.3. Otherwise, $U_{K}$ is an affine open subset of a smooth projective conic over $K$, not isomorphic to $\mathbb{A}_{K}^{1}$. So $U_{K}$ admits a Néron lft-model over $S$ by Proposition 7.7 .

Next we examine when the Néron lft-model is of finite type.

Proposition 7.11. Let $S$ be an excellent Dedekind scheme with field of functions $K$. Let $U_{K}$ be an affine smooth geometrically connected curve of $K$, not isomorphic to $\mathbb{A}_{K}^{1}$, and let $C_{K}$ be its regular compactification. Denote by $\Delta_{K}:=C_{K} \backslash U_{K}$. Let $C$ be a relatively minimal regular model of $C_{K}$ over $S$ and let $\Delta$ be the reduced Zariski closure of $\Delta_{K}$ in $C$. Let $U$ be the Néron lft-model of $U_{K}$ over $S$. Then the following properties are true.

(1) The scheme $U / S$ is of finite type if and only if $\Delta_{K}\left(K_{s}^{\mathrm{sh}}\right)=\emptyset$ for all closed points $s \in S$ and if $\Delta_{s} \cap C_{\mathrm{sm}, s}\left(k(s)^{\mathrm{sep}}\right)=\emptyset$ for almost all $s \in S$.

(2) Assume $S$ is infinite. For each closed point $s \in S$, set $U(s):=$ $U \times{ }_{S} \operatorname{Spec}\left(\mathcal{O}_{S, s}\right)$, the local Néron lft-model of $U_{K}$ over $\operatorname{Spec}\left(\mathcal{O}_{S, s}\right)$. Let $K^{\text {sep }}$ denote a separable closure of $K$.

(i) $\Delta_{K}\left(K^{\mathrm{sep}}\right)=\emptyset$ if and only if all the local Néron lft-models $U(s)$ are of finite type.

(ii) If $\Delta_{K}\left(K^{\mathrm{sep}}\right) \neq \emptyset$, the local Néron lft-models $U(s)$ are not of finite type for all but finitely many closed points $s \in S$. In particular, if $K$ has characteristic 0 , then $U$ is never of finite type.

Proof. (1) The $S$-scheme $U$ is of finite type if and only if

(a) for all closed point $s \in S, U(s)$ is of finite type over $\mathcal{O}_{S, s}$; and if

(b) $U$ is of finite type over some open dense subset of $S$, or equivalently, $U_{s}$ is connected for all but finitely many $s$ (Proposition 2.8 ).

Therefore we only need to show that the conditions of (1) are equivalent to the conditions (a) and (b) above.

First assume that $C_{K}$ is of arithmetic genus $>0$. Then $U_{K} \subseteq X_{K}:=$ $\operatorname{sm}\left(C_{K} / K\right)$. Let $X$ be the Néron model of $X_{K}$ over $S$, equal to the smooth locus $C_{\mathrm{sm}}$ of $C / S$ (Theorem 4.1). The minimal regular model $C$ commutes with strict henselization and completion ([8], 9.3.28), so by Theorem 5.1, for any closed point $s \in S, U(s)$ is of finite type over 
$\mathcal{O}_{S, s}$ if and only if $\Delta_{K}\left(K_{s}^{\mathrm{sh}}\right)=\emptyset$. Now consider the connectedness at a closed point $s \in S$. Shrinking $S$ if necessary, we can suppose $X_{s}=C_{\mathrm{sm}, s}$ is connected. If $\Delta_{s} \cap C_{\mathrm{sm}, s}\left(k(s)^{\mathrm{sep}}\right)=\emptyset$, then $U_{s}=X_{s} \backslash \Delta_{s}$ (Proposition 5.1 (3)) is connected. Conversely, suppose $U_{s}$ is connected. By separatedness, $U_{s} \rightarrow X_{s}$ is an open immersion. Then over $\mathcal{O}_{S, s}$, $U \rightarrow X$ is an open immersion, as $X \backslash U$ contains $\Delta_{K}$, hence $U \subseteq X \backslash \Delta$. By (5.1), we have $U=X \backslash \Delta\left(\right.$ over $\left.\mathcal{O}_{S, s}\right)$, thus $\Delta_{s} \cap C_{\mathrm{sm}, s}\left(k(s)^{\mathrm{sep}}\right)=\emptyset$ (Theorem $5.1(3)$ ).

Now suppose $C_{K}$ is a smooth conic. Let $s \in S$ be a closed point. If $\Delta_{K}\left(K_{s}^{\mathrm{sh}}\right)=\emptyset$, then $U(s)$ is of finite type over $\mathcal{O}_{S, s}$ by Proposition 6.1. Conversely, suppose $\Delta_{K}\left(K_{s}^{\mathrm{sh}}\right) \neq \emptyset$. It is enough to show $U(s)$ is not of finite type over $\mathcal{O}_{S, s}^{\text {sh }}$. Thus we can suppose $K$ strictly henselian and $\Delta_{K}(K) \neq \emptyset$. If there are two rational points in $\Delta_{K}$, then $U_{K}$ is isomorphic to an open subscheme of $\mathbb{G}_{m, K}$, hence $U(s)$ is not of finite type by Theorem 5.1 (1). Otherwise, there exists a non-rational point $q_{\infty} \in \Delta_{K}$. We apply again Theorem 5.1 (1) to $U_{K} \subset C_{K} \backslash\left\{q_{\infty}\right\}$ to conclude that $U(s)$ is not of finite type. So again $U(s)$ is of finite type if and only if $\Delta_{K}\left(K_{s}^{\mathrm{sh}}\right)=\emptyset$.

To see the equivalence between Condition (b) above and the second condition of (1), we are allowed to shrink $S$ and suppose that $C / S$ is smooth with connected fibers. The condition $\Delta_{s} \cap C_{\mathrm{sm}, s}\left(k(s)^{\mathrm{sep}}\right)=\emptyset$ then implies that $C \backslash \Delta$ is the Néron model of $U_{K}$ over $\mathcal{O}_{S, s}$ (see Claim in the proof of Proposition 6.1). Thus $U=C \backslash \Delta$ is of finite type with connected fibers over $S$. Conversely, suppose $U / S$ is of finite type (up to replace $S$ by some open dense subset). Shrinking $S$ if necessary, the isomorphism $U_{K} \rightarrow(C \backslash \Delta)_{K}$ extends to an isomorphism $U \cong C \backslash \Delta$. If there exists $x \in \Delta_{s} \cap C_{\mathrm{sm}, s}\left(k(s)^{\mathrm{sep}}\right)$, then there exists $y \in U_{K}\left(K_{s}^{\mathrm{sh}}\right)=$ $(C \backslash \Delta)_{K}\left(K_{s}^{\text {sh }}\right)$ which does not specializes to $U_{s}$. Contradiction with the Néron mapping property of $U$.

(2) As $S$ is infinite, $\Delta_{K}\left(K^{\text {sep }}\right) \neq \emptyset$ if and only if $\Delta_{K}\left(K_{s}^{\text {sh }}\right) \neq \emptyset$ for some (hence for almost all) $s \in S$. Thereby (2) follows directly from (1).

Remark 7.12 In general it is not true that if $\Delta_{K}\left(K^{\mathrm{sep}}\right)=\emptyset$ then $U$ is of finite type. One can construct examples similar to that of Oesterlé ([1], 10.1/11), showing that the existence of local Néron models does not imply the existence of global Néron model. Let $S$ be an excellent Dedekind scheme of characteristic $p>0$ with infinitely many closed points, such that each closed point of $S$ has perfect residue field (for example, take $S$ a smooth algebraic curve over a perfect field of characteristic $p$ ). Let $q_{\infty} \in \mathbb{P}_{K}^{1}$ be a purely inseparable closed point of degree $>1$. Let $U_{K}=\mathbb{P}_{K}^{1} \backslash\left\{q_{\infty}\right\}$ and let $U$ be its $S$-Néron model. 
Then $U \times{ }_{S} \operatorname{Spec}\left(\mathcal{O}_{S, s}\right)$ is of finite type. But as $U_{s}$ has two connected components for almost all $s \in S, U$ is not of finite type over $S$ by Proposition 2.8 (3).

\section{REFERENCES}

[1] S. Bosch, W. Lütkebohmert and M. Raynaud, Néron models, Ergebnisse der Math., 3. Folge, Bd. 21, 1990.

[2] T. Chinburg, Minimal models for curves over Dedekind rings, in Arithmetic Geometry edited by G. Cornell and J. H. Silverman. Springer-Verlag, 1986.

[3] B. Edixhoven, On Néron models, divisors and modular curves, J. Ramanujan Math. Soc. 13 (1998), 157-194.

[4] O. Gabber, Q. Liu and D. Lorenzini, Hypersurfaces in projective schemes and a moving lemma, preprint (2011).

[5] A. Grothendieck and J. Dieudonné, Éléments de géométrie algébrique, Publ. Math. IHÉS 4 (Chapter 0, 1-7, and I, 1-10), 8 (II, 1-8), 11 (Chapter 0, 8-13, and III, 1-5), 17 (III, 6-7), 20 (Chapter 0, 14-23, and IV, 1), 24 (IV, 2-7), 28 (IV, 8-15), and $\mathbf{3 2}$ (IV, 16-21), 1960-1967.

[6] S. Kleiman, The Picard scheme, Fundamental algebraic geometry, 235-321, Math. Surveys Monogr., 123, Amer. Math. Soc., Providence, RI, 2005.

[7] S. Lichtenbaum, Curves over discrete valuation rings, Amer. J. Math. 90 (1968), 380-405.

[8] Q. Liu, Algebraic geometry and arithmetic curves, Oxford Graduate Texts in Mathematics 6, Oxford University Press, paperback new edition (2006).

[9] Q. Liu and D. Lorenzini, Models of curves and finite covers, Compositio Math. 118 (1999), no. 1, 61-102.

[10] H. Matsumura, Commutative algebra (second edition), The Benjamin /Cummings Company, Inc., 1980.

[11] A. Néron, Modèles minimaux des variétés abéliennes sur les corps locaux et globaux, Publ. Math. IHES, 21 (1964), 128 pp.

[12] D. Popescu, General Néron desingularization and approximation, Nagoya Math. J., 104 (1986), 85-115.

[13] M. Raynaud: p-groupes et réduction semi-stable des courbes, The Grothendieck Festschrift, Vol. III, 179-197, Progr. Math., 88, Birkhäuser Boston, Boston, MA, 1990.

[14] M. Raynaud: Groupes vectoriels et schéma de Picard, C. R. Acad. Sci. Paris, Ser. I, 338 (2004), 223-227.

[15] Stacks Project Authors: Stacks Project, stacks.math.columbia.edu, (2013).

Université de Bordeaux 1, Institut de Mathématiques de Bordeaux, CNRS UMR 5251, 33405 TALEnce, France

E-mail address: Qing.Liu@math.u-bordeaux1.fr

E-mail address: Jilong.Tong@math.u-bordeaux1.fr 